\title{
Paving the way towards complex blood-brain barrier models using pluripotent stem cells
}

\author{
Lauschke, Karin; Frederiksen, Lise; Hall, Vanessa Jane
}

\section{Published in:}

Stem Cells and Development

Link to article, DOI:

$10.1089 /$ scd.2017.0003

Publication date:

2017

Document Version

Publisher's PDF, also known as Version of record

Link back to DTU Orbit

Citation $(A P A)$ :

Lauschke, K., Frederiksen, L., \& Hall, V. J. (2017). Paving the way towards complex blood-brain barrier models using pluripotent stem cells. Stem Cells and Development, 26(12). https://doi.org/10.1089/scd.2017.0003

\section{General rights}

Copyright and moral rights for the publications made accessible in the public portal are retained by the authors and/or other copyright owners and it is a condition of accessing publications that users recognise and abide by the legal requirements associated with these rights.

- Users may download and print one copy of any publication from the public portal for the purpose of private study or research.

- You may not further distribute the material or use it for any profit-making activity or commercial gain

- You may freely distribute the URL identifying the publication in the public portal 


\title{
Paving the Way Toward Complex Blood-Brain Barrier Models Using Pluripotent Stem Cells
}

\author{
Karin Lauschke, ${ }^{1,2}$ Lise Frederiksen, 3 and Vanessa Jane Hall ${ }^{4}$
}

\begin{abstract}
A tissue with great need to be modeled in vitro is the blood-brain barrier (BBB). The BBB is a tight barrier that covers all blood vessels in the brain and separates the brain microenvironment from the blood system. It consists of three cell types [neurovascular unit (NVU)] that contribute to the unique tightness and selective permeability of the BBB and has been shown to be disrupted in many diseases and brain disorders, such as vascular dementia, stroke, multiple sclerosis, and Alzheimer's disease. Given the progress that pluripotent stem cells (PSCs) have made in the past two decades, it is now possible to produce many cell types from the BBB and even partially recapitulate this complex tissue in vitro. In this review, we summarize the most recent developments in PSC differentiation and modeling of the BBB. We also suggest how patient-specific human-induced PSCs could be used to model BBB dysfunction in the future. Lastly, we provide perspectives on how to improve production of the BBB in vitro, for example by improving pericyte differentiation protocols and by better modeling the NVU in the dish.
\end{abstract}

Keywords: blood-brain barrier, induced pluripotent stem cell, in vitro model, neurodegenerative disease, scaffold, bioprinting

\section{Introduction}

$\mathrm{T}$ HE BLOOD-BRAIN BARRIER (BBB) is one of the three selective permeable barriers that protects the central nervous system from the peripheral system and forms at the interface of the microvasculature (arterioles, capillaries, and venules) of the brain and the brain parenchyma. The role of the BBB is multifactorial and includes diffusion of nutrients, removal of metabolites from the brain, preventing the entry of neurotoxic proteins into the brain and regulating leukocyte permeation [1]. It consists of a single layer of brain microvascular endothelial cells (BMECs), which line the blood vessels and interact closely with an outer encapsulating layer of pericytes, as well as astrocytes, neurons, and microglia. Together, these cell types form a complex cellular and physical interaction with surrounding cells and physical forces from the blood stream that regulate the BBB's permeable properties [2].

The BBB has been extensively studied, as it is an important barrier to permeabilize in the case of delivering drugs to the brain. However, it is affected and undergoes breakdown during normal aging [3], as well as in several brain disorders, including stroke [4] and epilepsy [5]. It is also perturbed in many neurodegenerative diseases, such as amyotrophic lateral sclerosis (ALS) [6], Parkinson's disease (PD) [7], Huntington's disease (HD) [8], vascular dementia [9], and Alzheimer's disease (AD) [10-13].

Modeling the $\mathrm{BBB}$ in a dish is a particularly attractive way to study the molecular mechanisms that may be involved in its breakdown; however, it is also useful for determining the transport of novel drugs through the barrier, before clinical trial testing. In vitro modeling traditionally uses cultured BMECs from varying species; however, these have limitations, including species differences [14] and low barrier tightness due to the monoculture of BMECs [15].

Building a complex BBB with its various cell types generated from human pluripotent stem cells (hPSCs) would be the ultimate in vitro model of the BBB and would be useful for determining mechanisms underlying disease/disorder. However, to date, there are only a limited number of studies that attempt to produce the BBB from PSCs. Here, we outline the current progress in the field and where the field is heading. We provide a background on the anatomical and molecular features of the BBB, as well as a brief overview of the in vitro cellular models in widespread use, before focusing on the PSC-derived BBB

\footnotetext{
${ }^{1}$ National Food Institute and ${ }^{2}$ Department of Micro- and Nanotechnology, Technical University of Denmark, Kongens Lyngby, Denmark.

${ }^{3}$ Faculty of Health and Medical Sciences, University of Copenhagen, København N, Denmark.

${ }^{4}$ Department of Veterinary and Animal Sciences, Faculty of Health and Medical Sciences, University of Copenhagen, Frederiksberg C, Denmark.
} 
research. We then consider how protocols could be improved by advancing cellular differentiation protocols, by using different matrices, both chemical and mechanical, and more complex cell culture models.

\section{Cell types of the BBB}

The monolayer of BMECs together with the encapsulating pericytes are ensheathed in an extracellular matrix (ECM), termed the basement membrane, and astrocytes project their end-feet toward this basement membrane (Fig. 1) [16]. The ratio between the endothelial cells (ECs), astrocytes, and pericytes in vivo is estimated to be 1:5:1 [17]. Other glia, such as oligodendrocytes and microglia, as well as neurons, also interact with these cells. A frictional force from the flowing blood along the vasculature system on the BMECs forms what is known as shear stress, one of the important physical factors controlling BBB permeability [16] (Fig. 1).

Brain microvascular endothelial cells. BMECs are specialized epithelial cells that form the physical neurovascular barrier through their effective tight junctions (TJs) (Fig. 2). BMECs are enclosed by pericytes with an estimated coverage of $30 \%$ [18], and both cell types lie on ECM proteins. Importantly, the pericytes and astrocytes regulate the BMECs and thereby BBB tightness, although it is only the pericytes that directly contact the BMECs.

The BMEC's TJs consist of a complex of proteins, including occludin and claudin, which span the membranes of two adjacent BMECs that hold them close together [16]. These proteins are often used as markers for barrier formation of BMECs. The formation of TJs occurs after the formation of another cell junction in the BMECs, the adherens junctions [19]. Similar to TJs, adherens junctions consist of a complex of proteins, including VE-cadherin and E-cadherin proteins, which span the membrane of two neighboring BMECs and are also useful markers for BBB tightness [16,20] (Fig. 2).
Pericytes. Pericytes exhibit multipotent properties that are similar to mesenchymal stem cells, and they have been demonstrated to differentiate into both osteoblasts and adipocytes. In the BBB, the absence of pericytes increases BBB permeability [21]. This is believed to be regulated by the secretion of various mediator molecules that affect transporter and TJ protein expression in the BMECs [22]. Pericytes also regulate the basement membrane by expressing and secreting components of the ECM, and they help direct astrocyte end-feet to the basement membrane [23]. Pericytes have a unique synergistic interaction with BMECs, due to their close contact, but further roles have yet to be identified due to the difficulty in isolating this cell type and also since it does not have a unique expression profile.

Astrocytes. The BMECs and pericytes are ensheathed by astrocytes, which contact the ECM with their end-feet, which has an estimated coverage of $~ 99 \%$ [18]. Astrocytes secrete mediator molecules from their end-feet to stimulate both pericytes and BMECs. Some of these secreted mediator molecules affect the expression of TJ and transporter proteins, whereas others, such as prostaglandin E or nitric oxide, stimulate pericytes to contract or relax to regulate blood flow [23]. Astrocytes are also activated as a result of inflammation and secrete pro-inflammatory mediators, which increases BBB permeability and leukocyte infiltration [24].

\section{Transport through the BBB}

The BBB facilitates passage across the BMECs in five alternate ways: (1) Paracellular transport occurs between the BMEC plasma cell membranes, through the TJs, allowing for transport of water-soluble agents. This is regulated by the TJs, restricts the diffusion of ions and other polar substances through the BBB, and blocks the passage of macromolecules [25] (Fig. 2). Importantly, the BMECs are highly polarized and contain a luminal and an abluminal side, which differ in lipid composition and transport proteins. This allows for transcellular passage of lipid-soluble

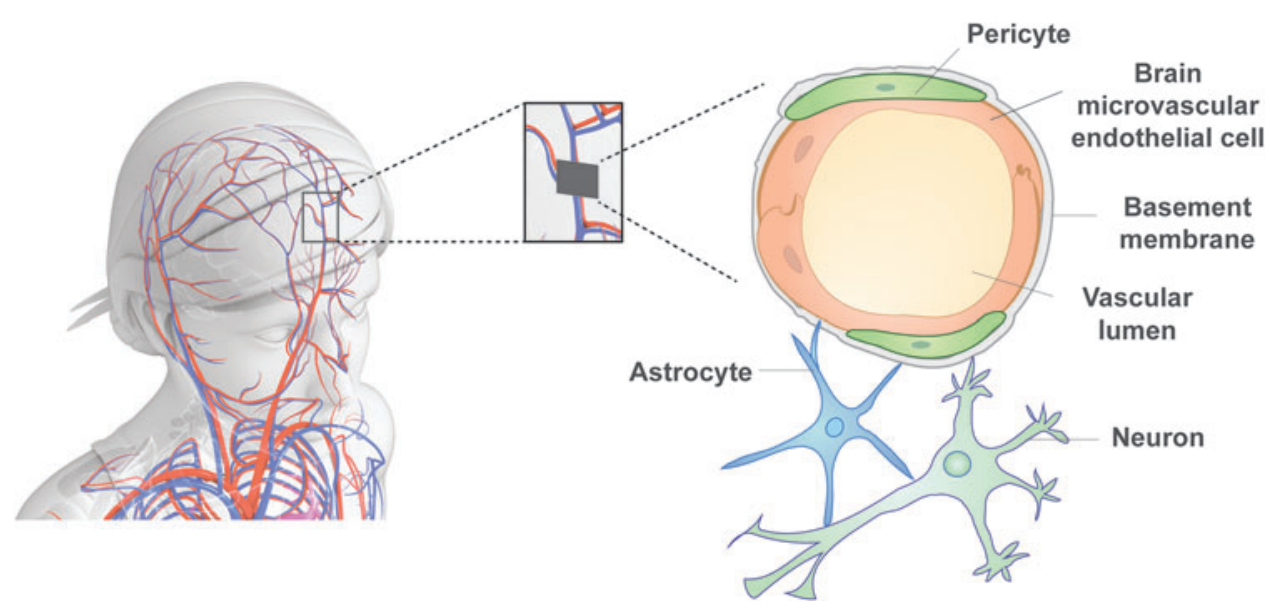

FIG. 1. The blood-brain barrier in vivo. The BBB is a tight and selective barrier that protects the brain from the peripheral system, which is permitted by the cell types of the NVU. BMECs form the epithelium of the blood vessels in the brain and are covered by pericytes, which cover about $30 \%$ of the endothelial cells. Both cell types contribute to and are unsheathed in the basement membrane. Astrocytes attach to the basement membrane with their end-feet, and neurons are in close proximity to the other cell types. Together, these cells form the NVU. BBB, blood-brain barrier; BMEC, brain microvascular endothelial cells; NVU, neurovascular unit. 


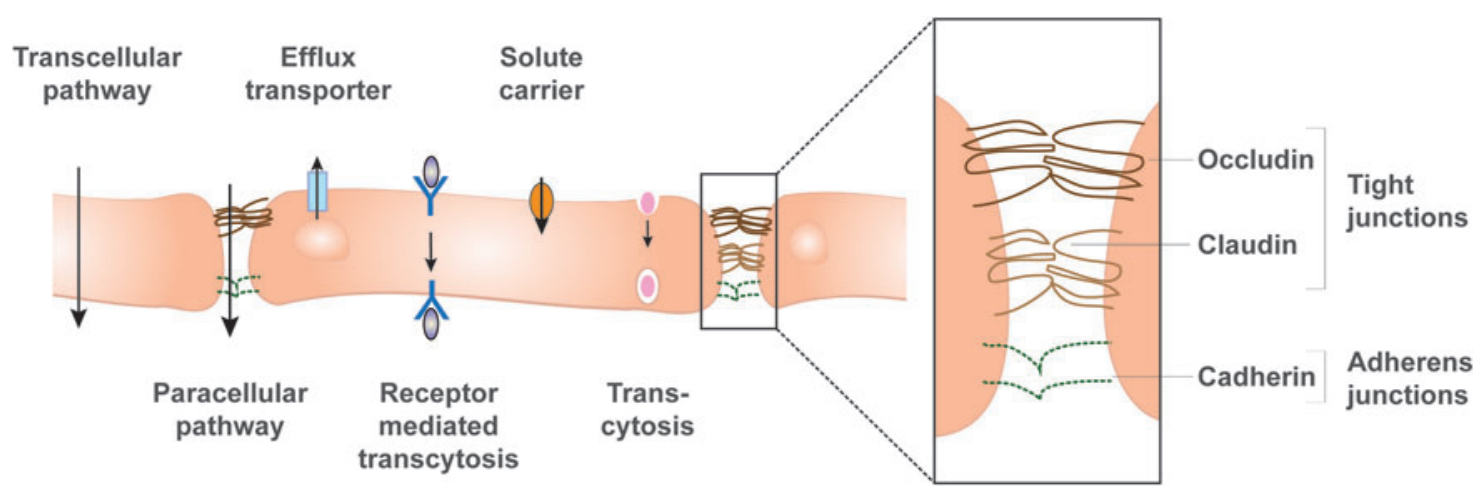

FIG. 2. Transport mechanisms through the BBB. The BMECs are connected by TJs and adherens junctions that facilitate the transcellular tightness. TJs consist of a complex of proteins, including occludin and claudin, whereas the main adherens junctions are cadherins. This tight connection between adjacent endothelial cells restricts paracellular transport, thereby making the BBB impermeable for small molecules and ions, which can be measured by transendothelial resistance. Transcellular transport is possible for lipid-soluble agents that pass through the cells by diffusion. Efflux transporters in the luminal membrane compartment, such as P-glycoproteins, actively transport molecules back into the vascular lumen. Specific receptors shuttle signaling molecules through the cells, and solute carriers transport nutrients from the lumen into the abluminal (parenchymal) side. Also, adsorptive transcytosis facilitates transport through the barrier. These pathways facilitate selective permeability and can be measured by the transport of proteins such as dextran or lucifer yellow. TJ, tight junction.

agents that can enter and pass through the BMECs by diffusion. (2) Proteins and nutrients may pass through the BMECs by specific solute carriers, such as the glucose transporter 1 (GLUT-1) [26]. A list of the varying BBB transporters can be reviewed [27]. (3) There are also efflux transporters such as P-glycoprotein (P-gp) and multidrug resistance protein 2 (MRP2) that actively transport a variety of molecules from the brain back into the bloodstream [16]. (4) Receptor-mediated transcytosis constitutes another mechanism that BMECs use to carry insulin and transferrin across the cell membrane. (5) Lastly, adsorptive transcytosis shuttles albumin and other plasma proteins directly through BMECs (Fig. 2).

Regardless of all these different transport mechanisms, the BMECs have a very limited permeability, which is particularly important, since it repels neurotoxic agents, but this also creates a challenge for finding drugs that can be delivered to the brain. The restriction of ions leads to a transendothelial electrical resistance (TEER), which can be measured experimentally and is a key parameter for assessing BBB tightness. The physiological TEER value in humans is estimated to be around $5,000 \Omega \mathrm{cm}^{2}$ (for further details, see Methods Used to Detect BBB Tightness section).

\section{Endothelial monoculture and co-culture models}

BMEC monocultures were the first in vitro BBB models developed (Fig. 3A). In the simplest setting, the cells are grown on a microporous filter membrane that separates a dish into two compartments, an apical and a basolateral side of the filter. In this model, BMECs have been used from various origins, that is, mouse, rat, pig, cow, monkey, or human (for a review of the models from different origins, see Helms et al. [27]). In the case of human BMECs, the availability of human primary BMECs is particularly restricted due to limited available post-mortem tissues. Therefore, immortalized human adult BMEC lines have been developed, for example, the widely used human cerebral endothelial cell line (hCMEC/D3)
[28] (for review, see Weksler et al. [29]). However, BMEC lines in monoculture de-differentiate and downregulate many genes involved in cell-cell adhesion and maturation compared with primary BMECs [30-32]. Low TJ tightness leads to unphysiologically low TEER values of maximum $300 \Omega \mathrm{cm}^{2}$ [29], potentially due to the lack of stimulating factors from other cell types [15]. To accommodate the other cell types of the neurovascular unit (NVU) in one setup, several co-culture models have been developed:

First, the co-culture of BMECs with astrocytes is reported in several different models (Fig. 3C). The experimental setup can vary, with the BMECs cultured on the upper side of a porous filter and (i) the astrocytes are grown on the lower side of the transwell filter (Fig. 3C), (ii) the astrocytes are grown on the bottom of the well (Fig. 3C), or (iii) BMECS are cultured with astrocyte-conditioned medium (set up as in Fig. 3A) [15].

Second, the addition of pericytes to BMECs alone, and to BMEC and astrocyte co-cultures, has also been attempted (Fig. 3B, D). Hatherell et al. describe a triple culture of human ECs, astrocytes, and pericytes in varying settings, as depicted in Fig. 3D [33]. One interesting study that included pericytes in its co-culture model detected reasonable TEER values of a maximum of $400 \Omega \mathrm{cm}^{2}$, when astrocytes were cultured on the bottom of the transwell, pericytes on the lower side of the filter, and ECs on the upper side of the filter [34]. This first triple-culture model also revealed expression of several marker proteins in the BMECs and a good correlation with in vivo permeability of characterized compounds [35].

Despite these positive outcomes, several studies have revealed opposing outcomes when triple cultures have been attempted [33,34,36,37]. Another very recent study has also systematically compared 10 different setups of mono- and co-cultures and found profound effects on the TEER value when ECs are co-cultured with both primary pericytes and human-induced pluripotent stem cells (hiPSC) derived neural stem cells (NSCs), or with primary pericytes, 
A

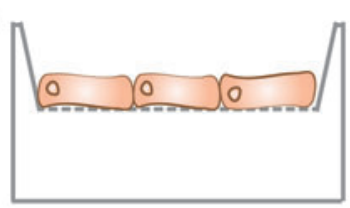

Monoculture

Weksler et al., 2005

Poller et al., 2008

Cechelli et al., 2014

Katt et al., 2016

D

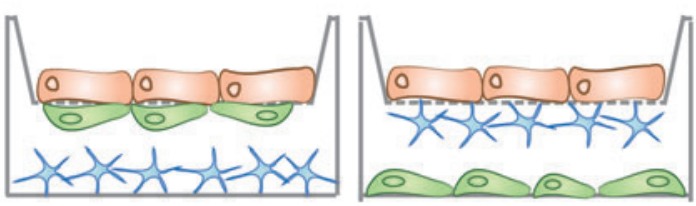

Triple-culture

Hatherell et al., 2011

$\mathbf{F}$

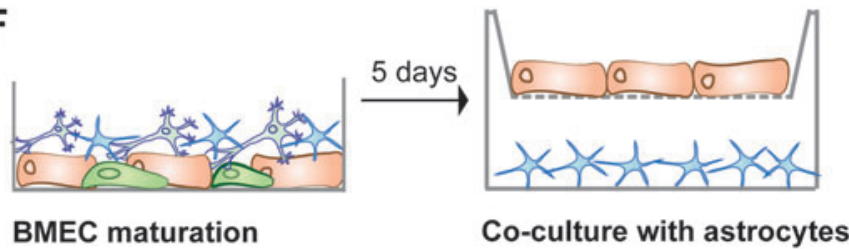

Yamamizu et al. 2017
B

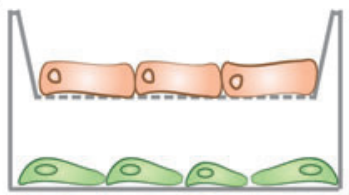

Co-culture with pericytes

Cechelli et al., 2014
C

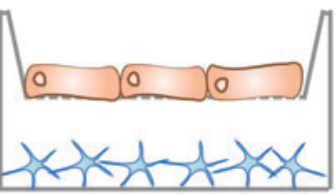

Co-culture with astrocytes

Lippmann et al., 2012

Di Ponio et al., 2014

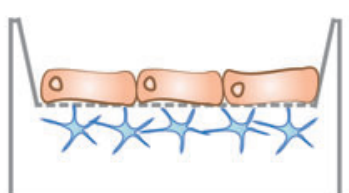

Hatherell et al., 2011

E

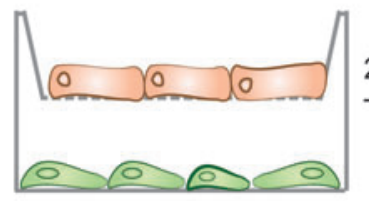

Pericyte priming

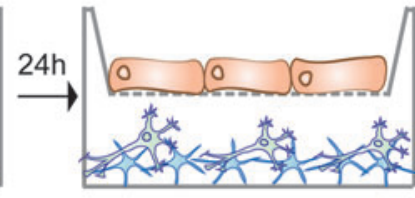

Co-culture with differentiated NPCs

Lippmann et al., 2014

FIG. 3. In vitro models of the BBB based on human BMECs. In the transwell system, several settings and combinations of the cell types of the NVU have been published: (A) Monoculture of BMECs grown on the upper side of the transwell filter. (B) Co-culture of BMECs grown on the upper side of the filter with pericytes on the bottom of the well. (C) Co-culture of BMECs grown on the upper side of the filter with astrocytes either on the bottom of the well or on the lower side of the transwell filter. (D) In the triple culture setting, BMECs are cultured with pericytes and astrocytes on the bottom of the transwell or the lower side of the filter. (E) BMECs on the upper side of the filter are first cultured with pericytes on the bottom of the well. After $24 \mathrm{~h}$, BMECs are cultured together with astrocytes and neurons, derived from NPCs, on the bottom of the well. (F) Co-culture of BMECs with PSC-derived pericytes, astrocytes, and neurons for 5 days, followed by BMEC maintenance on the upper side of the filter and astrocyte growth on the bottom of the transwell. NPC, neural progenitor cells.

astrocytes, and hiPSC-NSCs [38]. This study further supports the beneficial effects of pericytes on BBB tightness.

Lastly, the inclusion of neural progenitor cells (NPCs) into BMEC cultures (Fig. 3E) has been shown to increase BBB tightness in vitro [39]. This outcome has led to the further development of a co-culture model, including BMECs, NPCs (which develop into neurons), and astrocytes, which resulted in a BBB, reaching TEER values of a maximum of $250 \Omega \mathrm{cm}^{2}$ [39]. An overview of the current in vitro BBB models based on human BMECs can be found in Table 1.

\section{Generation and Characterization of In Vitro BBB Models}

\section{Methods used to detect BBB tightness}

To assess the integrity of the barrier in in vitro models, qualitative and quantitative methods have been established and will be presented here briefly: The characteristics of the TJ proteins, occludin, Zonula occludens 1 (ZO-1), and Zonula occludens 2 (ZO-2) can be imaged with freeze-fraction electron microscopy (FFEM) [40]. The functional tightness can be validated by the flux of molecules with different molecular weights such as dextran, lucifer yellow, sodium fluorescein, glucose, insulin, or mannitol. Another useful control is sucrose because it is BBB impermeable, hydrophilic and there is no brain-specific uptake mechanism [41]. To test for the expression of specific receptors or transporter proteins, these can also be selectively inhibited and the flux can be measured $[42,43]$. A widely used standard assay for permeability is the measurement of the TEER, which is given in $\Omega \mathrm{cm}^{2}$. For a concise review on TEER measurement techniques for in vitro models, we recommend reading the following article [44]. Importantly, it should be noted that the TEER can differ greatly depending on the experimental setup [44], and, therefore, independent studies cannot be directly compared.

\section{PSC differentiation into NVU cell types}

The recent advances in PSC technologies have opened new avenues toward generating more complex in vitro BBB 


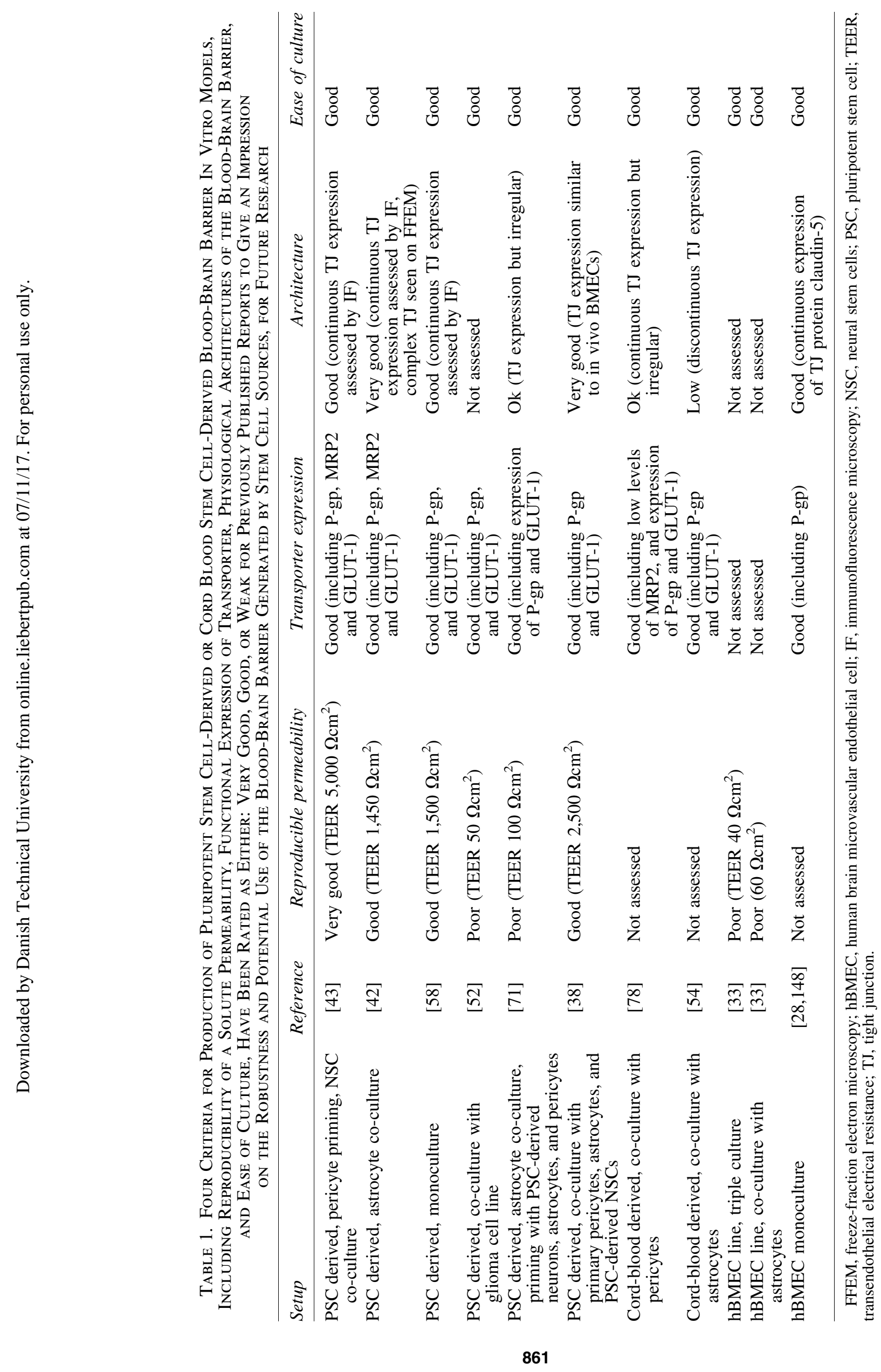


models, which are much closer to the in vivo situation in humans compared with the BMEC monocultures. Here, we discuss the progress in differentiation of the three main cell types of the NVU from PSCs that are summarized briefly in Table 2.

Brain microvascular endothelial cells. In general, ECs line the lumen of the microvasculature in many organs and there are profound tissue-specific characteristics of each EC type. In the case of BMECs, these are characterized by generating a high TEER and by releasing specific angiogenic factors [45]. It is, therefore, crucial to define markers for BMECs and to prove the BMEC identity on differentiation from PSCs. VE-cadherin and PECAM-1 are generally accepted markers for all ECs; however, the large heterogeneity of ECs among tissues hinders the use of one single marker for BMECs [46]. Therefore, a combination of tissue-specific markers and expressed proteins is needed to define BMECs as a unique EC, which should be combined with phenotypical assays, such as angiogenic or vasculogenic assays [47]. Therefore, we classify here that ECs are produced from PSCs rather than BMECs, unless proved otherwise by the authors.

Numerous protocols exist to generate ECs from hPSCs. These include spontaneous differentiation from embryoid bodies (EBs) [48] that can be improved by addition of growth factors for mesoderm induction, including basic fibroblast growth factor (bFGF) and insulin-like growth factor (IGF) together with vascular endothelial growth factor (VEGF) [49] or bone morphogenetic protein 4 (BMP4) [47]. Also, co-culturing of hPSCs [50] or hiPSCs [51] with the mouse bone marrow stromal cell line OP9 has yielded ECs. Improved protocols that lead to the derivation of BBBspecific BMECs include co-culturing with glioma cell lines [52], NPCs [53], or astrocytes [54].

These protocols rely on the same hypothesis, that coculture with neural cells pushes ECs toward a brain identity. The study by Minami et al. on co-culturing with a glioma cell line used CD34 and CD144 (VE-cadherin) as markers for ECs, which were sorted from the population and analyzed for CD31 (PECAM) and Von Willebrand factor to identify BMECs. This was further verified by detecting claudin, occludin, and ZO-1 expression, as well as by observing vascular-like structures in Matrigel ${ }^{\circledR}$ and uptake of acetylated low-density lipoprotein [52]. However, these cells alone yielded a TEER of only $55 \Omega \mathrm{cm}^{2}$, indicating a barrier tightness that is far from representative of the in vivo BMEC [52]. This indicated that induction by co-culturing with neural cultures is not sufficient for inducing BMEC properties, possibly due to the importance of factors secreted and/or mechanical interactions that pericytes and astrocytes have on BMECs.

In 2012, Lippmann et al. reported the first hiPSC-derived BMECs with $\mathrm{BBB}$ properties that could be produced with an efficiency of $>60 \%$ [42]. These cells were characterized by GLUT-1 and PECAM-1 expression, and their BBB identity was verified by staining for claudin-5, occludin, and VEcadherin. The authors attempted to mimic in vivo BBB development, where endothelial and neuronal lineages form at the same time. To this end, they kept hiPSCs in unconditioned medium without pluripotency factors, to allow for the differentiation of both neural and endothelial progenitors. After 5-7 days, this mixed population was switched to a medium that selectively stimulated EC proliferation for a further 1-2 days. The BMECs were then purified from the NPCs on selective collagen IV matrices, yielding a pure BMEC population (PECAM- $1^{+}$, GLUT- $1^{+}$cells). This population expressed BBB markers such as occludin and claudin5 , and it created a TEER of a maximum of $175 \Omega \mathrm{cm}^{2}$. On coculture with primary rat astrocytes grown on the bottom of the transwell, the monolayer of BMECs reached $412 \Omega \mathrm{cm}^{2}$.

This was further enhanced by optimizing the seeding density to create a more complete and an even monolayer that increased the TEER value further to $1,450 \Omega \mathrm{cm}^{2}$ [42]. In a follow-up study, the differentiation of hiPSCs was improved toward more physiological BMECs by the addition of retinoic acid (RA) to the EC medium. This led to increased expression of VE-cadherin, occludin, and claudin5 , as well as TEER values of more than $5,000 \Omega \mathrm{cm}^{2}$. Although the differentiation efficiency was not affected by the addition of RA, total cell proliferation was increased, leading to a higher number of BMECs $[43,55]$. RA is secreted by radial glia during brain development and is crucial for the development of a tight BBB during mouse development as well as in BMEC lines [56]. Within cells, it is bound by retinol-binding protein that is expressed in BMECs in vivo and also binds to the nuclear receptor STRA6 [57]. STRA6 expression is also upregulated during BMEC differentiation in vitro [42].

Recently, Katt et al. differentiated an hiPSC line into BMECs by using the Lippmann protocol with RAsupplemented EC medium, but they altered the timing of two steps in the protocol. The same markers were used as in the Lippmann study. Differentiation of hiPSC culture was initiated by changing to undefined medium without the pluripotency factor bFGF at $70 \%$ confluence. When the BMEC morphology was most prevalent (at day 6), the medium was changed to EC medium containing RA. This approach yielded a differentiation efficiency of an impressive $93 \%$ and a TEER of 2,000 $\Omega \mathrm{cm}^{2}$ [58]. Another study by Appelt-Menzel et al. reproduced the differentiation of BMECs with the Lippmann protocol and retrieved TEER values of a maximum of $2,500 \Omega \mathrm{cm}^{2}$ in a co-culture model with NSCs, astrocytes, and pericytes [38]. Importantly, FFEM revealed that the TJ proteins showed a similar morphology as reported on brain ECs in vivo, showing that morphologically relevant BMECs can be retrieved from hiPSCs [38].

In conclusion, the co-culture with neural cell types is promising to retrieve ECs with brain characteristics. However, the most critical step toward generating functional BMECs, which generate high TEER values, from stem cell sources is the use of a combination of astrocytes and EC medium supplemented with RA. Nevertheless, despite the apparent efficiency of the Lippmann protocol, there are no reports to date from other laboratories that can reproduce the reported TEER values of $5,000 \Omega \mathrm{cm}^{2}$.

Pericytes. Differentiation of PSCs into pericytes has not been as successful as differentiation into BMECs due to the lack of knowledge on the factors that produce pericytes in vivo. Furthermore, many pericyte markers are also expressed by ECs, mesenchymal cells, and smooth muscle cells, with the exception of the platelet-derived growth factor receptor (PDGFR $\beta$ ), and, therefore, characterization of pericytes in a dish is difficult. Pericytes have been created from PSCs by spontaneous differentiation followed by screening for pericyte-lineage markers and subsequent 


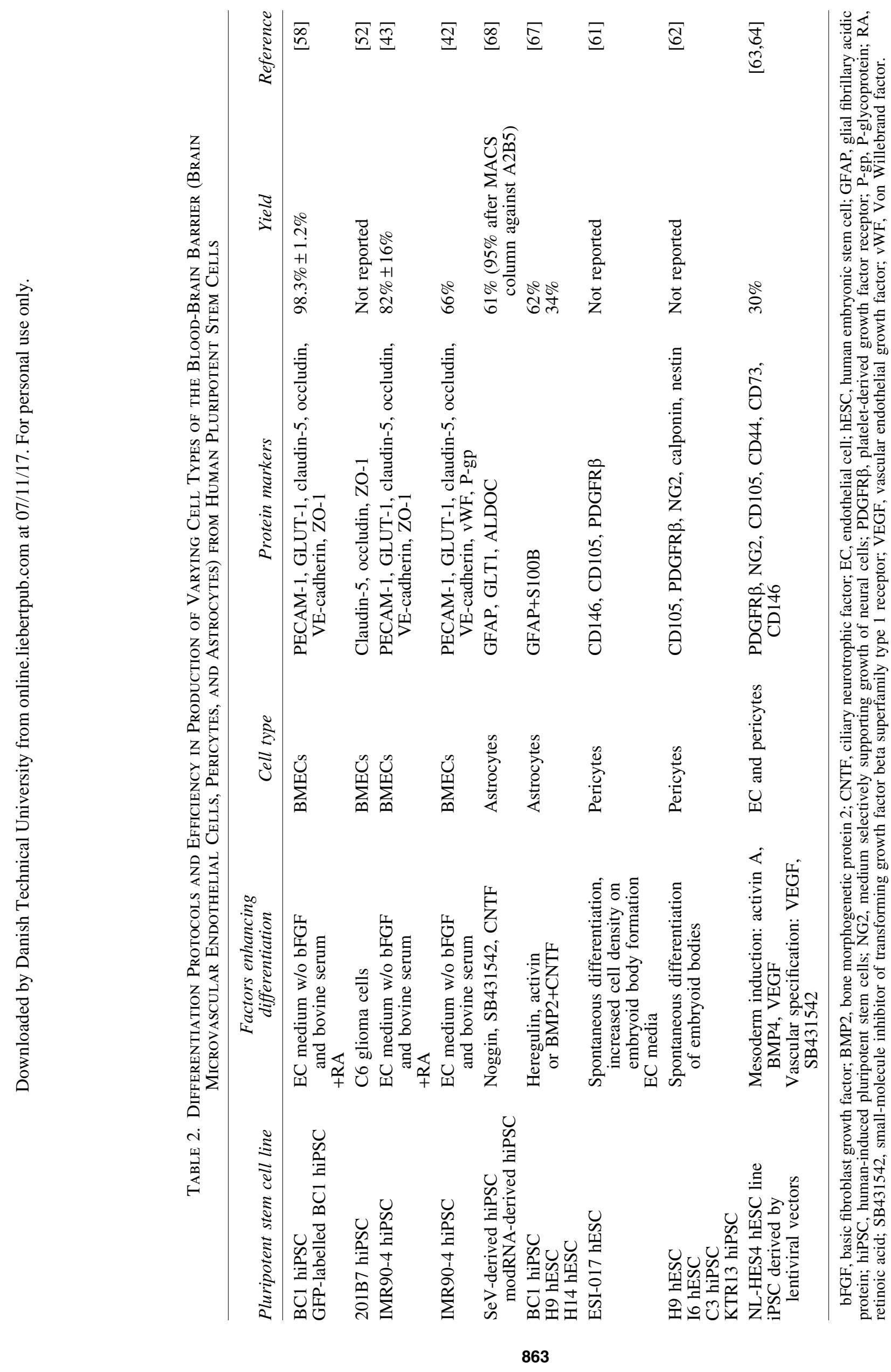


isolation of perivascular precursors. Differentiation into vascular cell types is enhanced by low oxygen conditions, which can be used to produce early vascular cells that can be further matured into pericytes and ECs [59,60]. A recent study found that increasing cell seeding density for spontaneous differentiation of EBs from PSCs resulted in cells with a mesenchymal-like morphology, which were supposedly perivascular precursors [61]. However, the cells were unable to differentiate into either osteoblasts or adipocytes, which is a hallmark feature of pericytes [61].

Another group applied a similar approach and continuously analyzed and isolated cells with pericyte-lineage markers during differentiation, without the use of selective media. After 7 weeks, isolated cells expressed several pericyte markers, including PDGFR $\beta$, and showed morphological features of human placenta pericytes that could differentiate into both adipogenic and osteogenic tissue [62]. Orlova et al. induced PSCs directly into the mesodermal lineage by inducing SMAD2/3 and WNT activation, followed by vascular specification by supplementing VEGF and inhibiting transforming growth factor- $\beta$. Cells expressing EC markers were isolated and expanded, and after 3 days pericytes appeared as a homogenous population of cells that were positive for pericyte markers, including PDGFR $\beta$. The study reported a pericyte differentiation efficiency of $30 \%$ defined by PDGFR $\beta$-positive cells $[63,64]$. Despite this progress, the differentiation of pericytes is still far from optimal and requires further improvement of differentiation protocols to increase the percentage of cells, which we discuss in the next section on improvements of BBB production in vitro.

Astrocytes. Contrary to the other BBB cell types, astrocyte differentiation from PSCs is well established and astrocytes have been successfully differentiated since 2011 [65], although the initial protocols were relatively long (6 months) $[65,66]$. However, a more recent protocol for producing astrocytes was successful after only 5 weeks. It employs an initial differentiation step into NPCs followed by activation of the JAK/STAT and SMAD pathways to activate the astrocytespecific glial fibrillary acidic protein (GFAP) gene. This protocol resulted in an efficiency of 60\%-80\%, defined by GFAP [67]. Another study by Mormone et al. used initial SMAD inhibition in their protocol followed by JAK/STAT activation and reported the generation of $60 \%$ GFAP-positive astrocytes. To enhance the purity of the astrocytes, the cells were then magnetic-activated cell sorted by using the astrocyte marker A2B5, which resulted in a purity of 95\% GFAP-positive astrocytes $[68,69]$.

\section{Complex in vitro PSC-derived BBB models}

To date, only two laboratories have published multicellular in vitro models of the BBB from hiPSCs. Lippmann et al. gained human BMECs from hiPSCs as discussed earlier $[42,43]$, and they combined these in a multicellular BBB model with human neurons and astrocytes generated from NPCs [43]. hiPSC-derived BMECs were co-cultured first with primary human pericytes for $24 \mathrm{~h}$ and, subsequently, with a mixture of astrocytes and neurons differentiated from primary human NPCs (Fig. 3E). This optimal culture condition led to TEER values of more than $5,000 \Omega \mathrm{cm}^{2}$, whereas culture with only pericytes reached slightly lower levels of $4,500 \Omega \mathrm{cm}^{2}$ [43]. Culture without pericytes only reached half of the TEER values, supporting the importance of pericytes in BBB tightness. Intriguingly, this group advanced their model by employing astrocytes, neurons, and BMECs from the same hiPSC source [70]. hiPSC-derived BMECs were co-cultured with neurons and astrocytes, which were produced through EZ spheres from the same hiPSC source. The monoculture of hiPSC-derived BMECs reached a TEER value of $200 \Omega \mathrm{cm}^{2}$, whereas the co-culture with the isogenic neurons and astrocytes (in a ratio 1:3) elevated the TEER to nearly $800 \Omega \mathrm{cm}^{2}$ [70]. This was slightly lower than in the previously reported co-culture with NPC-derived astrocytes and neurons $\left(1,450 \Omega \mathrm{cm}^{2}\right)$ [42]. However, the isogenic coculture model did not include pericytes, which might increase the TEER values, as previously presented in the model with pericyte priming (up to $5,000 \Omega \mathrm{cm}^{2}$ ) [43].

The second laboratory published an interesting approach, deriving all four cell types of the BBB from hiPSC (Fig. 3F) [71]. The group previously characterized that cyclic AMP efficiently supports the differentiation of hiPSC toward ECs [72-75]. However, as in the other studies, co-culture with pericytes, astrocytes, and neurons is still necessary to induce BMEC hallmarks [71]. Strikingly, for their co-culture, they used hiPSC-derived neurons, astrocytes, and pericytes. The EC and pericytes were differentiated together by inducing first mesoderm formation and then the vascular endothelial lineage, followed by sorting ECs and pericytes by combined cell surface markers. Astrocytes and neurons were derived from EBs over a period of 120 days.

Having generated all four cell types, the researchers combined them into one culture for 5 days to induce BMEC hallmarks in the ECs. However, not all four cell types were transferred to a transwell BBB model. Only the BMECs and astrocytes were transferred (Fig. 3F). Unfortunately, TEER values of only $100 \Omega \mathrm{cm}^{2}$ were achieved [71], which might potentially be increased by the further addition of pericytes and neurons into the transwell system. The beneficial effect of pericytes was also found in a systematic comparison of different multicellular models, in which hiPSC-BMECs were cocultured with hiPSC-derived or primary neuronal cells and pericytes [38].

These studies highlight many advantages of hPSCs to create in vitro models of the BBB: First, BMEC can be obtained easily in high quantities due to efficient differentiation protocols. Second, the co-differentiation experiments with NPCs [42,43], the isogenic co-culture model [76], and the fully PSC-derived model [71] showed that, in principle, all BBB cell types can be made from the same stem cell source. Third, the obtained BMECs showed extremely high TEER values that have never been generated with any other BMEC source. The PSC-derived BMECs have also been shown to respond well in artificially invoked stress conditions, which might be valuable for some assays. For example, PSC-derived BMECs have been reported to respond more physiologically to hypoxia than the hCMEC/D3 cell line [77]. Most importantly, however, is that a combination of co-culturing with varying (primary) cell sources appears to be a significant advantage of improving hiPSC-derived BMEC BBB tightness [38].

There have also been studies describing the production of BBB from other stem cell sources, that is, cord bloodderived stem cells in a co-culture with either pericytes [78] or astrocytes [54]. However, we believe that hiPSCs represent a superior stem cell source since: (1) hiPSCs are a 
renewable source with very little ethical concerns attached; (2) hiPSCs are easy to manufacture, and numerous hiPSC lines are available; and (3) patient-derived hiPSCs might recapitulate the disease phenotype in vitro.

\section{What do we expect from an ideal BBB model?}

An important step in generating a more physiologically relevant model of the human $\mathrm{BBB}$ is to ask: Which criteria should an ideal model fulfil? Although the criteria might differ depending on the experimental question, the model should mimic the in vivo BBB as close as possible. We highlight four points that could be adopted for estimating the value of an in vitro BBB model [79]: (1) reproducibility of a restrictive solute permeability (measured by the TEER and tracer flux); (2) functional expression of transporters (assessed by the efflux ratio of known molecules as well as specific inhibitors of known transporters); (3) physiologically realistic architecture containing polarity, that is, containing a luminal and an abluminal side, gross cell dimensions, surface area of interactions, as well as complexity of TJs (measured by light, fluorescence, and transmission electron microscopy or FFEM); and (4) easy to culture with acceptable cell proliferation rates and continuous growth [15]. In Table 1, we rate the previously published PSC-derived BBB according to how well they may fulfil these criteria and how physiologically relevant they are (Table 1$)$.

\section{Using PSCs for modeling neurovascular disease}

To date, several in vitro and in vivo models of neurovascular diseases exist, particularly from the mouse. However, there is a need for more human models that recapitulate the in vivo disease state. hPSC-derived neural models have particularly flourished in recent years, since they can recapitulate different neural cell types readily. However, models of the diseased BBB are needed, since it is affected in many neurological diseases. Moreover, there is a need to include the BBB together with PSC-derived neural cells since these models could help to more closely recapitulate the physiological and physical interactions that might lead to the detection of disease phenotypes that have not been recapitulated in vitro yet, for example, neuronal death that might only be induced by BBB breakdown [80].

Disease-causing mutations often cause phenotypes in only certain cell types, or regions, as, for example, in ALS, where only motor neurons and glial cells are affected, which has been successfully recapitulated with ALS patient-derived hiPSCs [81]. Also, other neurological syndromes are highly cell-type specific, such as AD, PD, and HD. Selective loss of neurons in these diseases leads to impaired cognitive and mobile function, depending on the site and severity of neural loss [82]. Not long after iPSCs were first produced came the first publication of iPSCs generated from patients with neurofibromatosis type 1 disease [83]. Since then, several proof-of-principle studies have shown that both sporadic and genetic diseases can be modeled with hiPSCs from patients [84].

For example, PD has been modeled with hiPSC-derived dopaminergic neurons carrying mutations such as a triplication of synuclein-alpha (SNCA) [85] or the most common mutation in LRRK2 [86], both of which lead to increased levels of $\alpha$ synuclein. Another study found altered ERK signaling in derived dopaminergic neurons that, when repressed, was able to reverse a certain disease pathology, such as sensitivity to oxidative stress and neurite shortening [87]. Also, proteasome dysfunction, oxidative stress, and increased expression of $\alpha$-synuclein have been observed in PARK2 mutant hiPSC-derived neurons [88]; however, no Lewy body formation or neurodegeneration has been recapitulated in hiPSC-derived dopaminergic neurons. Also, AD $[89,90]$ and HD [91] have been mimicked in the dish and reproduced important disease phenotypes. However, none of the neurons die in culture, as seen in AD patients [8,92]. Recently, it was reported that healthy neurons from hiPSC degenerate if transplanted in PD model mouse brains, suggesting that amyloid plaques from the diseased environment are responsible for neural degeneration [93].

Intriguingly, the permeability of the BBB has been shown to be altered in these diseases, although it is not clear whether this is a cause or a consequence of the disease [94,95]. BBBrelated mutations, as, for example, in the $A B C B 1$ gene, encoding the P-gp efflux transporter expressed in BMECs, have been associated with PD [96,97]. Similarly, BBB breakdown has also been seen in AD patients [98]. Furthermore, important BMEC transporters such as GLUT-1 and P-gp have been found with impaired function in $\mathrm{AD}$ patients [10,99].

Remarkably, the APOE protein, of which isoform 4 (APOE4) is a major risk factor for AD, is expressed in the endfeet of astrocytes [100]. Deletion of APOE in mice, in fact, leads to BBB breakdown, as displayed by increased leakage of dye into the brain of APOE-deficient mice compared with wild-type mice [101], and this difference increases with age [102]. Moreover, replacement of the endogenous APOE gene in mice with the human $\mathrm{AD}$ risk factor isoform $A P O E 4$ impairs BBB function [103]. Later, these results were confirmed by multiphoton microscopy of fluorescence-conjugated dextran, which was widely distributed in the brains of mice expressing deletion mutants or APOE4 [95]. This elegant study further revealed that fluorescently labeled neurotoxins accumulate in neurons of these mice, suggesting an increased neurotoxin exposure through the leaky BBB.

Importantly, at the time of BBB breakdown ( 2 weeks of age), neuronal activity measured by voltage-sensitive dyes was the same in mutant and wild-type mice, whereas it was dramatically decreased in mutant mice at 4 months of age [95]. This substantiates the theory that $\mathrm{BBB}$ breakdown precedes neuronal changes and underpins the in vivo relevance of a tight $\mathrm{BBB}$.

These studies highlight the possibility that BBB dysfunction might be causal or an early feature of $A D$, and that the $\mathrm{BBB}$ is, therefore, important to be included in an in vitro disease model. Also, for HD, the disease hallmark, that is, accumulation of the mutant Huntington (mhtt) protein in various cell types of the brain, has also been found in cells of the BBB in both mouse models and post-mortem tissue from HD patients [8]. Importantly, protein levels of the TJ-proteins claudin-5 and occludin were lower in mouse models of HD [8] and AD [95], and this correlated with a larger intracellular space between adjacent BMECs in HD mice [8].

To summarize, hiPSC have been successfully employed to model neurovascular diseases, although not all disease phenotypes have been recapitulated in vitro. Emerging evidence suggests that $\mathrm{BBB}$ disintegrity is involved in these diseases, and, therefore, the inclusion of BBB cell types in these models might potentially aid in fully exposing all disease phenotypes. Retrieval of the BBB cell types from 
hiPSCs is particularly attractive since the neurovascular cell types cannot be easily derived from human patients, unless under surgery, for example, if a tumor is excised. Therefore, a promising alternative is to generate each cell type from the BBB by using hiPSCs derived from the patient.

\section{Future Considerations for Improving BBB Production In Vitro}

For future BBB models, one should consider the tremendous improvements in cell culture and tissue engineering that also enhance the differentiation of stem cells. Stem cell fate is influenced exclusively by its extracellular chemical and physical environment, which is termed the "stem cell niche," a concept first proposed in 1978 by Schofield [104]. In vivo, this is also a place of residence for the stem cells, in addition to its surrounding microenvironment that controls their differentiation and self-renewal [105]. Stem cells, therefore, require a mechanical substrate to grow on, as well as soluble factors that regulate their fate. Important factors of the niche in vivo may be secreted from other cells, the ECM, paracrine factors, and metabolites [106]. Many of these characteristics require recapitulation in vitro, and this can partly be achieved by using innovative (bio-) materials and microfabrication techniques to modify the culture environment systematically [107].

All the current PSC-derived BBB models lack an important feature, which is blood flow. Blood flow is an important physiological factor influencing the BBB in vivo, by activating mechanosensors on the apical surface of the epithelium [108]. This physical stimulus has been proved to regulate EC differentiation and adhesion [109] as well as cell division, apoptosis, cell migration, and polarization of the epithelium [110]. It stimulates TJ expression and cell adhesion in primary and immortalized BMECs [110] such as ZO-1, claudin-5 [111,112], and the efflux transporter P-gp [112]. Drug transporters and metabolizing enzymes are expressed at higher levels under shear stress induced by blood flow [113]. Also, increased in vitro shear stress has been demonstrated to improve permeability values and to induce differentiation of ESCs into endothelial-like cells [114,115].

Side-by-side comparisons with bovine EC lines cultured with or without shear stress of 4 dynes $/ \mathrm{cm}^{2}$ in hollow fibers showed an increase in TEER from $60 \Omega \mathrm{cm}^{2}$ to more than $600 \Omega \mathrm{cm}^{2}$ [116]. This was confirmed in a recent study on rat primary BMECs, where TEER values rose fourfold to 1,300 $\Omega \mathrm{cm}^{2}$. In this study, cells were grown in a three-dimensional (3D) microfluidic device directly on a collagen bed, with medium flow applied through a medium channel on the cells [117]. The increased barrier tightening is believed to involve the adherens junction protein VE-cadherin, by signaling the shear stress response to occludin and changing TJ protein expression and organization [111].

Nevertheless, the effects of shear stress on multicellular BBB models and on iPSC-derived ECs have yet to be analyzed.

Historically, flow has been created in hollow fibers by a computer-controlled pump to reach values between 5 and 23 dynes $/ \mathrm{cm}^{2}$, which is similar to in vivo values [110]. However, there are drawbacks of the hollow fiber system. These include its unsuitability for high-throughput screening and that the cells cannot be analyzed morphologically in the tube. Therefore, alternative ways to employ shear stress are required to intro- duce flow of the medium in perfusion culture dishes during or after differentiation, potentially through use of microfluidic systems. For example, flow might be employed through inlet and outlet channels that push liquid through a hydrogel reservoir in which the cells are cultured [118]. Alternatively, cells might be grown on a membrane within a microfluidic device with a flow channel on top of the cells, as described by Booth and Kim in 2014 [119]. Importantly, shear stress might act on only one side of the ECs (apical or basal), and the flow can be used to transport nutrients and waste products as well.

\section{Three-dimensional culturing}

It has been observed that 3D scaffolds provide physiologically more relevant models and are closer to in vivo models than conventional two-dimensional (2D) cell cultures [120]. Threedimensional scaffolds support the differentiation of stem cells into desired cell types and tissues, for example, in culturing neurons and epithelial cells. For example, a remarkable step in the development of brain organoids was accomplished with the use of Matrigel as a supportive 3D matrix [121].

Besides the choice of scaffold material, several possibilities exist for the 3D setup of the BBB depending on the purpose of the model. For drug delivery and safety studies, the major focus lies on permeability of the barrier, and the developed models, therefore, must contain two compartments that are separated by a filter membrane. On the contrary, for phenotypical or molecular analysis, this is not necessary and simpler models are sufficient.

For example, a multicellular spheroidal BBB model arranged all cell types of the BBB in direct contact without the need of a surrounding scaffold; primary human BMECs, pericytes, and astrocytes self-assembled spontaneously into a tube-like structure [122]. Although the barrier tightness and therefore functionality of this BBB model could not be assessed, it is still fascinating that the cells assembled spontaneously into layers with BMECs forming an outer monolayer, astrocytes accumulating in the lumen of the spheroid, and pericytes forming a layer between the other two cell types [122].

Lastly, 3D models without two compartments can still be used to study the uptake of molecules into BMECs or other cell types of the BBB. Although this gives no information on permeability values measured by TEER, it might give an indication as to whether a substance can enter or cross the BMECs. For example, one study assessed the uptake of gold nanoparticles into BMECs or into 3D cultured astrocytes, which were covered by a layer of BMECS, by electron microscopy [123].

To introduce compartmentalization in 3D cultures, an elegant approach is to embed human astrocytes and pericytes into collagen type I and inject this mixture around needles into a chip, as developed by Tourovskaia et al. in 2014 [124]. After gelation, the needles are removed, leaving hollow channels in which hBMECs are injected to form a monolayer covering the walls of the channels. Tourovskaia et al. showed that in their setup, the pericytes and astrocytes in the collagen matrix grew in close contact with the BMEC monolayer, mimicking the in vivo situation closely. This led to a tight barrier, as assessed by imaging fluorescently labeled sucrose and dextran [124]. However, no TEER measurements were possible in this setting and the expression of $\mathrm{TJ}$ or transporter proteins was not analyzed, giving very little information on the physiology of the BBB model. 
Another strongly expanding field is the use of hollow fiber tubes to mimic the cerebrovasculature in $3 \mathrm{D}$, and to facilitate co-culture of cells as well as flow conditions in the tube $[125,126]$. However, the measurement of TEER and permeability requires more sophisticated setups that are compatible with the fiber architecture, compared with simple transwell systems. Nevertheless, several studies showed the feasibility of a commercially available hollow fiber system that includes electrodes covering the hollow fibers to measure the TEER. In this system, human BMECs were cocultured with human astrocyte cell lines in a hollow fiber and TEER values greater than $700 \Omega \mathrm{cm}^{2}$ were measured [127]. Also, within the area of microfabrication, several attempts to mimic the BBB have been pursued. This technology is reviewed in detail by Helm et al. [128].

Lastly, we envision further development of the classical transwell system (Fig. 3) by adding 3D neural tissues in either compartment. This may enhance the maturation of BMECs and the formation of physiological BBB characteristics, such as TJ formation and transporter expression. This could potentially be accomplished by 3D printing of the cells (bioprinting) to enhance an even distribution of cells across the scaffold. The feasibility of this approach has been recently demonstrated with NPCs embedded in a polyurethane (PU) dispersion [129]. The cell-laden dispersion was 3D printed into several layers of fibers and maintained at $37^{\circ} \mathrm{C}$, and the viability of the cells was proved after 2 days of culture [129]. Three-dimensional bioprinting with all cell types of the BBB could also potentially arrange all cell types in the same compartment, overcoming the limitations of spatial separation by a transwell filter. Importantly, this would also facilitate cell-cell interactions, which improves stem cell differentiation $[130,131]$. This might lead to formation of more physiological morphology and BBB tightness (Fig. 4A). We envision these techniques would further advance the recently developed BBB models, although this currently lies at the very edge of our technological abilities.

\section{Progress in bioengineering of scaffolds}

Introduction of better cell culture matrices and improved ECM substrates into these BBB models might help to improve culture conditions and may enhance the physiological matrix stiffness and shear stress [132]. In recent years, advanced scaffolds and biomimetic materials have been developed that facilitate a more controlled microenvironment
A

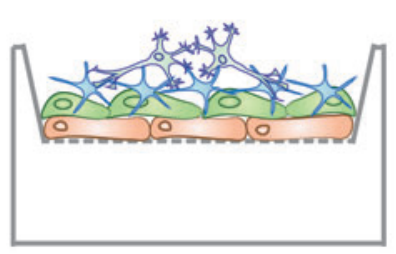

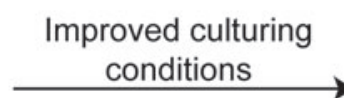

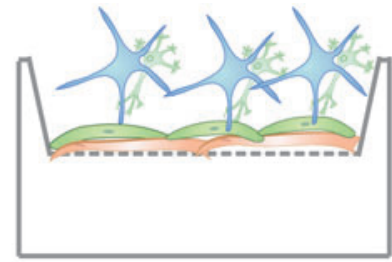

B

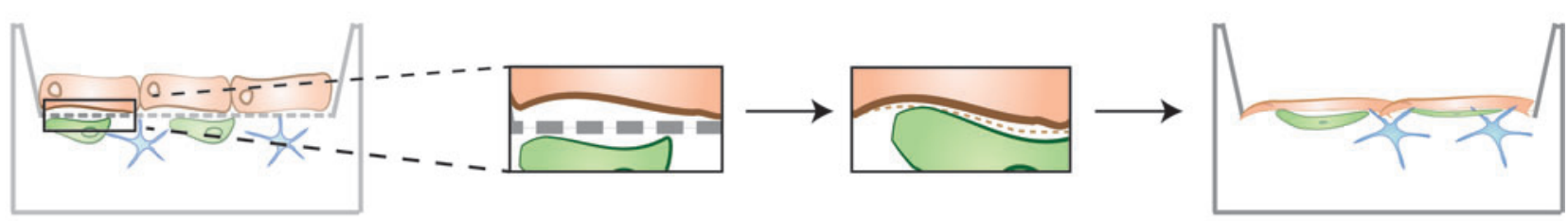

C

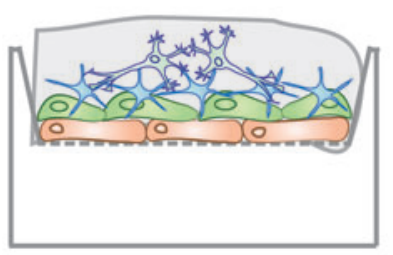

\section{Improved culturing} conditions
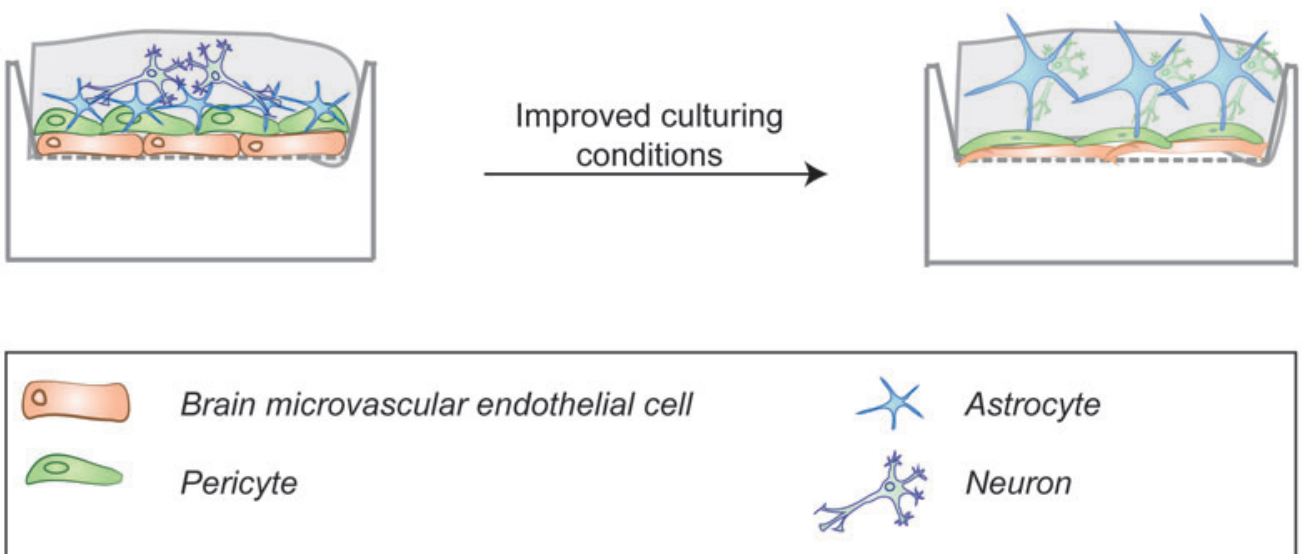

FIG. 4. Future considerations for improving BBB production from stem cell sources in vitro. Developments that might improve the traditional transwell filter systems. (A) Arranging all cell types of the NVU in the same compartment, potentially by 3D bioprinting (left). Through enhanced cell-cell interactions, this might lead to a more physiological cell morphology and BBB characteristics (right). (B) Novel materials might replace traditional filter membranes to reduce the physical separation between co-cultured cells. Biodegradable materials to produce the filter membrane might be used as well, and eventually degrade over time, to enable formation of more physiological-like barriers. (C) Cells might be encapsulated in 3D gels, such as hydrogels, that mimic the in vivo extracellular matrix more closely (left) and enhance cell morphology and barrier tightness during culture (right). 3D, three dimensional. 
in vitro [106]. We envision several applications where novel biomaterials might be applied:

First, novel materials might help to overcome certain limitations of the materials used for the filter membranes in transwell in vitro BBB models. One limitation in traditional transwell filters is the filter membrane with $10-20 \mu \mathrm{m}$ thickness, which well exceeds the size of the basal lamina and, therefore, acts as a physical barrier between the cells seeded on either side of the filter. These filter membranes could be replaced by membranes made from novel biomaterials, for example, biodegradable polymers, to provide an initial substrate for adherence, which, eventually, degrades later in culture to produce a more physiological boundary (Fig. 4B).

Second, novel scaffolds for 3D culturing or improved differentiation of hiPSC could be developed. Today, the most abundantly used 3D scaffold is Matrigel, which has been recently shown to improve BMEC monolayer barrier tightness [133]. Matrigel is commonly used as a scaffold to culture and differentiate hPSCs into BMECs, which are then seeded onto permeable transwell filter membranes that are coated with collagen and fibronectin [42,43].

Alternative scaffolds for $3 \mathrm{D}$ cultures can be constructed by the use of various polymers, hydrogels, silicons, and many more [106]. The choice of material depends greatly on the application and cell type, and the chosen material should mimic the in vivo supportive tissue. A special need in BBB modeling is that the scaffolds should enable the measurement of TEER values and, therefore, enable free movement of ions [134]. For example, collagen type I has been used in BBB models to embed the BBB cell types in a $3 \mathrm{D}$ gel $[124,135]$. Also, hyaluronic acid is often used in engineering brain tissue because it is also found in brain ECM and might be a suitable candidate to aid BBB models [134].

Polymers with many promising features for use in BBB modeling are hydrogels, since they mimic the ECM closely, are biocompatible and easy to fabricate. Various microfabrication techniques are available to produce 3D hydrogel scaffolds for tissue engineering [106]. Hydrogels can be produced from naturally derived polymers such as collagen, gelatin, algenate, or Matrigel, or synthetic polymers such as polylactic acid or PU. The latter has recently been used in bioengineering due to its good physical properties and biocompatibility [106].

Synthetic polymers bear the advantage of being chemically defined, reproducible, and modifiable in regards to their physical and chemical properties. However, these polymers are usually formed based on cross-linking chemistry, which is toxic for cells and, therefore, a problem if the cells are to be encapsulated in the hydrogel [136]. Recently, a technique was developed to produce hydrogels from PU dispersions without the need of cross-linking, which can, therefore, be used to encapsulate cells [129]. This technique might enable the wrapping of all three BBB cell types in the same scaffold, thereby improving the maturation and development of a physiological barrier (Fig. 4C).

Another advantage of hydrogels is that they can be loaded with chemicals, mimicking ECM-bound regulatory factors in vivo $[129,137]$. The ECM is known to provide important cues on cells both through its structure and its release of stimulating factors [138]. The use of more physiologically relevant ECM proteins is, therefore, important to consider, and the incorporation of additional ECM components might help to improve the functional properties and maturation of BMECs in vitro [139]. For example, ECM ligands secreted by ECs include not only fibronectin and collagen IV but also laminin, SPARC, Nidogen-1, and Agrin [140]. Recently, an elegant study showed that the composition and assembly of endogenous ECMs contribute to BBB formation in vitro [133].

Improvement of the scaffolds and where the cells are deposited could help to increase the contact between the different cell types as well as improve BMEC polarity, physiological stiffness, and the transport of metabolites and soluble regulators. Therefore, future BBB models could contain scaffolds that are loaded with ECM factors or use conditioned scaffolds that are similar to conditioned medium. Another important factor is the rigidity and elasticity of the scaffold. Bone cells need a rigid environment, whereas neurovascular cells require more elastic environments that are similar to the in vivo situation. Here, the composition of synthetic hydrogels could be varied to adjust the stiffness of the scaffold to the needs of the cells [136].

\section{Future perspectives for creation of PSC-derived BBB models}

There are several aspects that could be applied to improve differentiation of BMECs from PSCs. The cell density before the differentiation of PSCs has an effect on the resulting yield and TEER, and the optimal seeding density varies depending on the PSC line used [141]. Undefined sources such as Matrigel and feeder cells provide inconsistency, and alternatives of defined origin should be considered. The addition of RA has proved to significantly enhance barrier tightness as well as cell proliferation, leading to high numbers of BMECs [43]. Although the protocols for differentiating neurons and astrocytes are well established, and BMECs can be differentiated with a high efficiency, only few protocols exist for pericytes. This is partially due to a lack of pericyte markers; however, even without a definitive marker, pericytes can be characterized by their ability to form stable vascular tubes together with ECs [142]. This feature might be exploited, and also eventually co-culture of both cell types might improve the differentiation and maturation of pericytes.

The BBB models produced from PSC do not contain aged cells. Importantly, differentiated PSCs are more representative of cells from young, rather than aged individuals. This may be an issue if it is important to study diseases that have BBB breakdown in aged people.

The production of cell types from young individuals in vitro is likely due to the rejuvenation of cells after iPSC reprogramming, which results in increased telomere length $[143,144]$ and ESC-like mitochondria in the undifferentiated iPSCs [145]. Therefore, it could be important to mimic old age in the dish by accelerating the aging of hiPSC-derived cells. To this end, the differentiated cells can be exposed to stress paradigms, including toxins or other compounds that lead to oxidative or mitochondrial stress [146].

One intriguing method that has been used for aged iPSCderived cells in vitro is to overexpress progerin, a truncated isoform of laminin A, which is associated with premature aging. Overexpression in neurons that were differentiated from PD-derived iPSCs revealed age-related disease phenotypes that were otherwise not detected in vitro [147]. Based on these findings, it has been proposed that accelerating the 
aging of iPSC-derived cells could help to elucidate aging mechanisms coupled with the disease [146].

\section{Conclusion}

In conclusion, the greatest potential of PSCs for biomedical research lies in their ability to form complex tissues with phys- iologically relevant characteristics. PSCs have been able to yield several cell types of the $\mathrm{BBB}$, which is the important vascular interface between the brain and the blood, and it is affected in many neurodegenerative diseases and blood disorders. To date, the $\mathrm{BBB}$ has been modeled in relatively simple $2 \mathrm{D}$ cultures that are composed of monolayers of BMECs, yielding few physiological characteristics. However, the production of a more
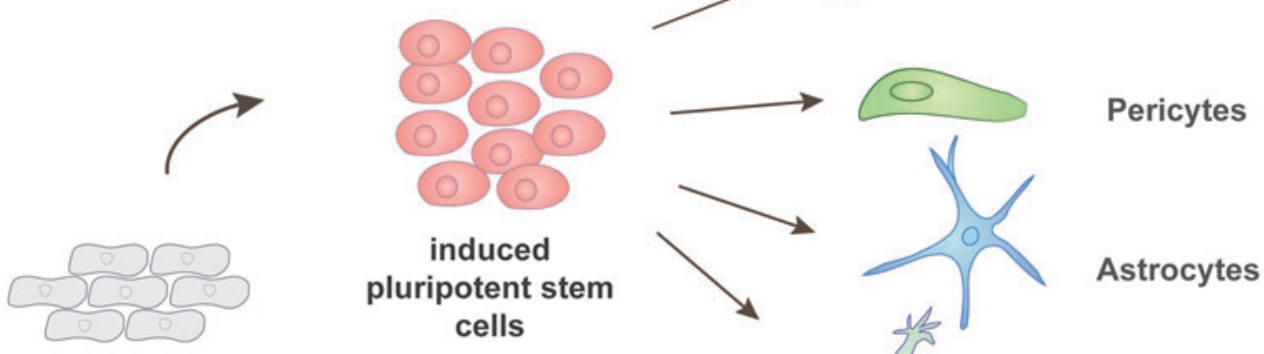

fibroblasts
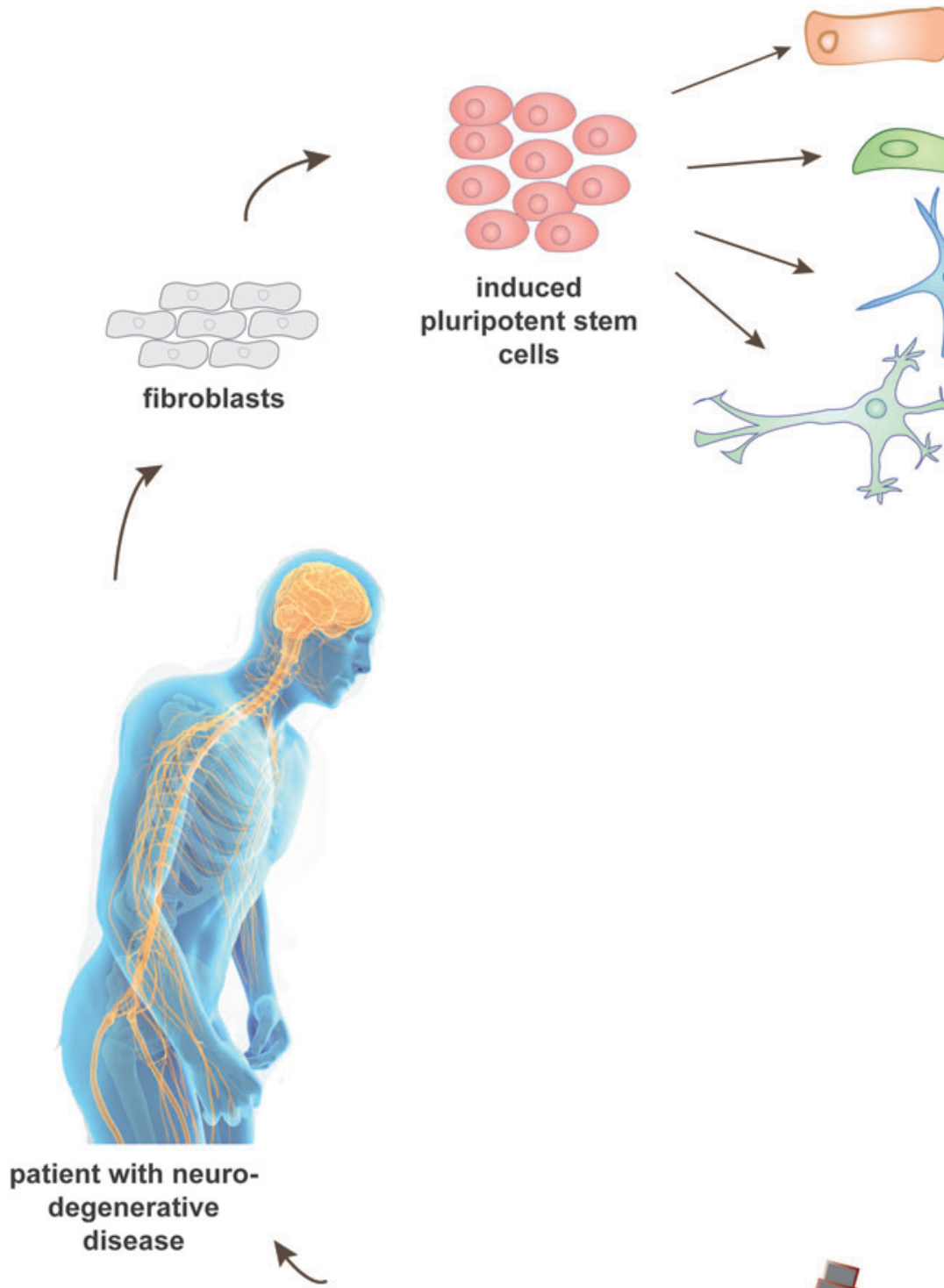

BMECS

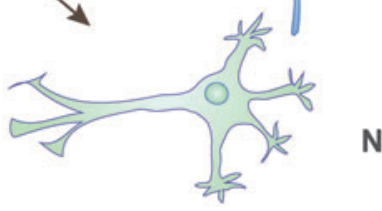

Neurons
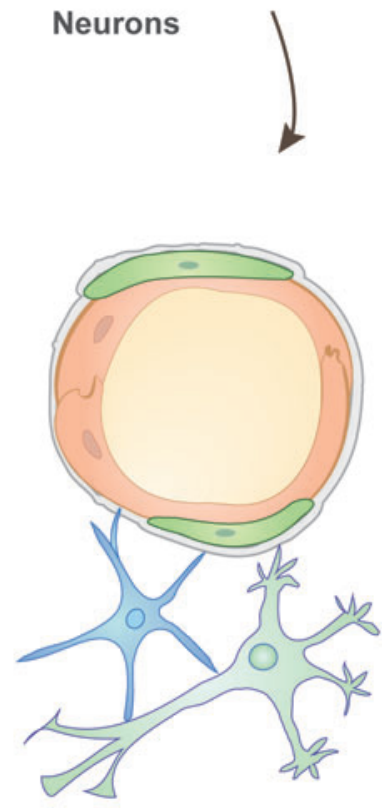

in vitro

blood-brain barrier model

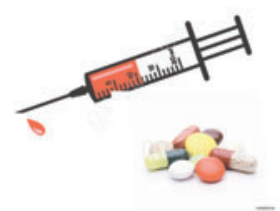

novel treatments

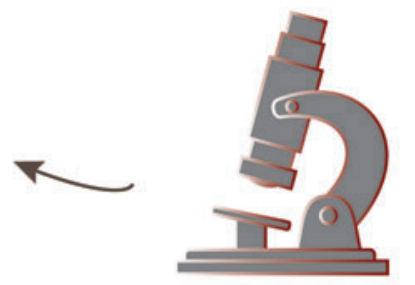

disease studies or drug development

FIG. 5. Future outlook for use of advanced BBB models for treatment of neurodegenerative diseases. Patients with neurovascular diseases donate their cells, for example, fibroblasts, for reprogramming into pluripotent stem cells. From these, all cell types of the NVU may be generated, that is, BMECs, pericytes, astrocytes, and neurons, to build advanced in vitro models of the BBB. These models can be used for studying the molecular mechanisms of the disease or for testing and developing novel drugs. This might also be useful for personalized medicine, that is, to find the best treatment for the respective patient. From such studies, novel treatments might evolve to improve life quality and survival of the patient. BMEC, brain microvascular endothelial cell; BBB, blood-brain barrier; NVU, neurovascular units. 
complex BBB, which contains several cell types of the NVU, would certainly recapitulate the physiological tightness and permeability better. The use of PSCs in creating this more complex BBB has been initiated, and great success in recreating the tightness has already been achieved. However, more complex cultures, the advances in biomaterials, and 3D culturing will definitely help to lead this field into even more advanced in vitro models of the BBB. Importantly, use of patient-specific hiPSCs to create the BBB in the future will help to determine more about the mechanisms related to BBB breakdown and provide more insight into disease mechanisms that might ultimately lead to the development of new drugs (Fig. 5).

\section{Acknowledgments}

The authors are grateful to Prof. Jenny Emnéus for reading the article and providing valuable input on bioengineering and scaffolds. V.J.H.'s current research and activities are funded by the Innovation Fund Denmark supported project, BrainStem (a Stem Cell Center of Excellence in Neurology).

\section{Author Disclosure Statement}

No competing financial interests exist.

\section{References}

1. Abbott NJ and A Friedman. (2012). Overview and introduction: the blood-brain barrier in health and disease. Epilepsia 53:1-6.

2. Sweeney MD, S Ayyadurai and BV Zlokovic. (2016). Pericytes of the neurovascular unit: key functions and signaling pathways. Nat Neurosci 19:771-783.

3. Lucke-Wold BP, AF Logsdon, RC Turner, CL Rosen and JD Huber. (2014). Chapter fourteen-aging, the metabolic syndrome, and ischemic stroke: redefining the approach for studying the blood-brain barrier in a complex neurological disease. Adv Pharmacol 71:411-449.

4. Merali Z and A Kassner. (2015). Assessment of bloodbrain barrier disruption in stroke. Stroke 46:3310-3315.

5. Marchi N, M Banjara and D Janigro. (2016). Blood-brain barrier, bulk flow, and interstitial clearance in epilepsy. $\mathrm{J}$ Neurosci Methods 260:118-124.

6. Winkler EA, JD Sengillo, JS Sullivan, JS Henkel, SH Appel and BV Zlokovic. (2013). Blood-spinal cord barrier breakdown and pericyte reductions in amyotrophic lateral sclerosis. Acta Neuropathol 125:111-120.

7. Korczyn AD. (2015). Vascular parkinsonism - characteristics, pathogenesis and treatment. Nat Rev Neurol 11:319-326.

8. Drouin-Ouellet J, SJ Sawiak, G Cisbani, M Lagacé, W-L Kuan, M Saint-Pierre, RJ Dury, W Alata, I St-Amour, et al. (2015). Cerebrovascular and blood-brain barrier impairments in Huntington's disease: potential implications for its pathophysiology. Ann Neurol 78:160-177.

9. Iadecola C. (2013). The pathobiology of vascular dementia. Neuron 80:844-866.

10. Montagne A, SR Barnes, MD Sweeney, MR Halliday, AP Sagare, Z Zhao, AW Toga, RE Jacobs, CY Liu, et al. (2015). Blood-brain barrier breakdown in the aging human hippocampus. Neuron 85:296-302.

11. Montagne A, DA Nation, J Pa and MD Sweeney. (2016). Brain imaging of neurovascular dysfunction in Alzheimer's disease. Acta Neuropathol 131:687-707.
12. Snyder HM, RA Corriveau, S Craft and JE Faber. (2015). Vascular contributions to cognitive impairment and dementia including Alzheimer's disease. Alzheimer's Dement 11:710-717.

13. Sweeney MD, AP Sagare and BV Zlokovic. (2015). Cerebrospinal fluid biomarkers of neurovascular dysfunction in mild dementia and Alzheimer's disease. J Cereb Blood Flow Metab 35:1055-1068.

14. Uchida Y, S Ohtsuki, Y Katsukura, C Ikeda, T Suzuki, J Kamiie and T Terasaki. (2011). Quantitative targeted absolute proteomics of human blood-brain barrier transporters and receptors. J Neurochem 117:333-345.

15. Krizbai IA and I Wilhelm. (2014). In vitro models of the blood-brain barrier for the study of drug delivery to the brain. Mol Pharm 11:1949-1963.

16. Abbott NJ, AAK Patabendige, DEM Dolman, SR Yusof and DJ Begley. (2010). Structure and function of the blood-brain barrier. Neurobiol Dis 37:13-25.

17. Pardridge WM. (1999). Blood-brain barrier biology and methodology. J Neurovirol 5:556-569.

18. Mathiisen TM, KP Lehre, NC Danbolt and OP Ottersen. (2010). The perivascular astroglial sheath provides a complete covering of the brain microvessels: an electron microscopic 3D reconstruction. Glia 58:1094-1103.

19. Tietz S and B Engelhardt. (2015). Brain barriers: crosstalk between complex tight junctions and adherens junctions. J Cell Biol 209:493-506.

20. Vorbrodt AW and DH Dobrogowska. (2003). Molecular anatomy of intercellular junctions in brain endothelial and epithelial barriers: electron microscopist's view. Brain Res Rev 42:221-242.

21. Armulik A, G Genové, M Mäe, MH Nisancioglu, E Wallgard, C Niaudet, L He, J Norlin, P Lindblom, et al. (2010). Pericytes regulate the blood-brain barrier. Nature 468:557561.

22. Hurtado-Alvarado G, AM Cabañas-Morales and B Gómez-Gónzalez. (2014). Pericytes: brain-immune interface modulators. Front Integr Neurosci 7:80.

23. Keaney J and M Campbell. (2015). The dynamic bloodbrain barrier. FEBS J 282:467-479.

24. Argaw AT, L Asp and J Zhang. (2012). Astrocyte-derived VEGF-A drives blood-brain barrier disruption in CNS inflammatory disease. J Clin Invest 122:2454-2468.

25. Wolburg H, S Noell, A Mack, K Wolburg-Buchholz and P Fallier-Becker. (2009). Brain endothelial cells and the glio-vascular complex. Cell Tissue Res 335:75-96.

26. Daneman R and A Prat. (2015). The blood-brain barrier. Cold Spring Harb Perspect Biol 7:a020412.

27. Helms HC, NJ Abbott, M Burek, R Cecchelli, P-O Couraud, MA Deli, C Förster, HJ Galla, IA Romero, et al. (2016). In vitro models of the blood-brain barrier: an overview of commonly used brain endothelial cell culture models and guidelines for their use. J Cereb Blood Flow Metab 36:862-890.

28. Weksler BB. (2005). Blood-brain barrier-specific properties of a human adult brain endothelial cell line. FASEB J 19:1872-1874.

29. Weksler B, IA Romero and P-O Couraud. (2013). The hCMEC/D3 cell line as a model of the human blood brain barrier. Fluids Barriers CNS 10:16.

30. Calabria AR and EV Shusta. (2008). A genomic comparison of in vivo and in vitro brain microvascular endothelial cells. J Cereb Blood Flow Metab 28:135-148.

31. Lyck R, N Ruderisch, AG Moll and O Steiner. (2009). Cultureinduced changes in blood-brain barrier transcriptome: impli- 
cations for amino-acid transporters in vivo. J Cereb Blood Flow Metab 29:1491-1502.

32. Urich E, SE Lazic, J Molnos, I Wells and PO Freskgård. (2012). Transcriptional profiling of human brain endothelial cells reveals key properties crucial for predictive in vitro blood-brain barrier models. PLoS One 7:e38149.

33. Hatherell K, PO Couraud, IA Romero and B Weksler. (2011). Development of a three-dimensional, all-human in vitro model of the blood-brain barrier using mono-, co-, and tri-cultivation transwell models. J Neurosci Methods 199:223-229.

34. Nakagawa S, MA Deli, S Nakao and M Honda. (2007). Pericytes from brain microvessels strengthen the barrier integrity in primary cultures of rat brain endothelial cells. Cell Mol Neurobiol 27:687-694.

35. Nakagawa S, Á Kittel, H Kawaguchi, T Shimono, K Tanaka, M Niwa, T Shimizudani and MA Deli. (2009). A new blood-brain barrier model using primary rat brain endothelial cells, pericytes and astrocytes. Neurochem Int 54:253-263.

36. Vandenhaute E, L Dehouck, M-C Boucau, E Sevin, R Uzbekov, M Tardivel, F Gosselet, L Fenart, R Cecchelli and M-P Dehouck. (2011). Modelling the neurovascular unit and the blood-brain barrier with the unique function of pericytes. Curr Neurovasc Res 8:258-269.

37. Hayashi K, S Nakao, R Nakaoke, S Nakagawa, N Kitagawa and M Niwa. (2004). Effects of hypoxia on endothelial/pericytic co-culture model of the blood-brain barrier. Regul Pept 123:77-83.

38. Appelt-Menzel A, A Cubukova, K Günther, F Edenhofer, J Piontek, G Krause, T Stüber, H Walles, W Neuhaus and M Metzger. (2017). Establishment of a human blood-brain barrier co-culture model mimicking the neurovascular unit using induced pluri- and multipotent stem cells. Stem Cell Reports 8:1-13.

39. Lippmann ES and C Weidenfeller. (2011). Blood-brain barrier modeling with co-cultured neural progenitor cellderived astrocytes and neurons. J Neurochem 119:507-520.

40. Severs NJ. (2007). Freeze-fracture electron microscopy. Nat Protoc 2:547-576.

41. Clark PA, AJ Al-Ahmad, T Qian, RR Zhang, HK Wilson, JP Weichert, SP Palecek, JS Kuo and EV Shusta. (2016). Analysis of cancer-targeting alkylphosphocholine analogue permeability characteristics using a human induced pluripotent stem cell blood-brain barrier model. Mol Pharm 13:3341-3349.

42. Lippmann ES, SM Azarin, JE Kay, RA Nessler, HK Wilson, A Al-Ahmad, SP Palecek and EV Shusta. (2012). Derivation of blood-brain barrier endothelial cells from human pluripotent stem cells. Nat Biotechnol 30:783-791.

43. Lippmann ES, EV Shusta, SM Azarin, SP Palecek and A Al-Ahmad. (2014). A retinoic acid-enhanced, multicellular human blood-brain barrier model derived from stem cell sources. Sci Rep 4:4160.

44. Srinivasan B, AR Kolli, MB Esch and HE Abaci. (2015). TEER measurement techniques for in vitro barrier model systems. J Lab Autom 20:107-126.

45. Wilson HK, SG Canfield, EV Shusta and SP Palecek. (2014). Concise review: tissue-specific microvascular endothelial cells derived from human pluripotent stem cells. Stem Cells 32:3037-3045.

46. Nolan DJ, M Ginsberg, E Israely, B Palikuqi, MG Poulos, D James, B-S Ding, W Schachterle, Y Liu, et al. (2013). Molecular signatures of tissue-specific microvascular en- dothelial cell heterogeneity in organ maintenance and regeneration. Dev Cell 26:204-219.

47. Rufaihah AJ, NF Huang, S Jamé, JC Lee, HN Nguyen, B Byers, A De, J Okogbaa, M Rollins, et al. (2011). Endothelial cells derived from human iPSCS increase capillary density and improve perfusion in a mouse model of peripheral arterial disease. Arterioscler Thromb Vasc Biol 31:e72-e79.

48. Levenberg S, JS Golub and M Amit. (2002). Endothelial cells derived from human embryonic stem cells. Proc Natl Acad Sci U S A 99:4391-4396.

49. Yu J, NF Huang, KD Wilson, JB Velotta, M Huang, Z Li, A Lee, RC Robbins, JP Cooke and JC Wu. (2009). nAChRs mediate human embryonic stem cell-derived endothelial cells: proliferation, apoptosis, and angiogenesis. PLoS One 4:e7040.

50. Vodyanik MA, JA Thomson and II Slukvin. (2006). Leukosialin (CD43) defines hematopoietic progenitors in human embryonic stem cell differentiation cultures. Blood 108:2095-2105.

51. Choi K-D, J Yu, K Smuga-Otto, G Salvagiotto, W Rehrauer, M Vodyanik, J Thomson and I Slukvin. (2009). Hematopoietic and endothelial differentiation of human induced pluripotent stem cells. Stem Cells 27:559-567.

52. Minami H, K Tashiro, A Okada, N Hirata and T Yamaguchi. (2015). Generation of brain microvascular endothelial-like cells from human induced pluripotent stem cells by co-culture with C6 glioma cells. PLoS One 10:e0128890.

53. Weidenfeller C, EV Shusta and CN Svendsen. (2007). Differentiating embryonic neural progenitor cells induce blood-brain barrier properties. J Neurochem 101:555-565.

54. Boyer-Di Ponio J, F El-Ayoubi, F Glacial, K Ganeshamoorthy, C Driancourt, M Godet, N Perrière, O Guillevic, PO Couraud and G Uzan. (2014). Instruction of circulating endothelial progenitors in vitro towards specialized blood-brain barrier and arterial phenotypes. PLoS One 9:e84179.

55. Stebbins MJ, HK Wilson, SG Canfield, T Qian, SP Palecek and EV Shusta. (2016). Differentiation and characterization of human pluripotent stem cell-derived brain microvascular endothelial cells. Methods 101:93-102.

56. Mizee MR, D Wooldrik, KA Lakeman, B van Het Hof, JA Drexhage, D Geerts, M Bugiani, E Aronica, RE Mebius, et al. (2013). Retinoic acid induces blood-brain barrier development. J Neurosci 33:1660-1671.

57. Kawaguchi R, J Yu, J Honda, J Hu, J Whitelegge, P Ping, P Wiita, D Bok and H Sun. (2007). A membrane receptor for retinol. Science 315:820-826.

58. Katt ME, ZS Xu, S Gerecht and PC Searson. (2016). Human brain microvascular endothelial cells derived from the BC1 iPS cell line exhibit a blood-brain barrier phenotype. PLoS One 11:e0152105.

59. Kusuma S and A Facklam. (2014). Characterizing human pluripotent-stem-cell-derived vascular cells for tissue engineering applications. Stem Cells Dev 24:451-458.

60. Kusuma S and S Gerecht. (2016). Derivation of endothelial cells and pericytes from human pluripotent stem cells. Methods Mol Biol 1307:213-222.

61. Greenwood M. (2016). A novel lineage restricted, pericyte-like cell line isolated from human embryonic stem cells. Sci Rep 6:24403.

62. Dar A, H Domev, O Ben-Yosef, M Tzukerman, N ZeeviLevin, A Novak, I Germanguz, M Amit and J Itskovitz- 
Eldor. (2012). Multipotent vasculogenic pericytes from human pluripotent stem cells promote recovery of murine ischemic limb. Circulation 125:87-99.

63. Orlova VV, Y Drabsch, C Freund, S Petrus-Reurer, FE van den Hil, $S$ Muenthaisong, $P$ Ten Dijke and CL Mummery. (2014). Functionality of endothelial cells and pericytes from human pluripotent stem cells demonstrated in cultured vascular plexus and zebrafish xenografts. Arterioscler Thromb Vasc Biol 34:177-186.

64. Orlova VV, FE van den Hil, S Petrus-Reurer, Y Drabsch, $\mathrm{P}$ ten Dijke and CL Mummery. (2014). Generation, expansion and functional analysis of endothelial cells and pericytes derived from human pluripotent stem cells. Nat Protoc 9:1514-1531.

65. Krencik R, JP Weick, Y Liu and ZJ Zhang. (2011). Specification of transplantable astroglial subtypes from human pluripotent stem cells. Nat Biotechnol 29:528-534.

66. Chandrasekaran A, HX Avci and M Leist. (2016). Astrocyte differentiation of human pluripotent stem cells: new tools for neurological disorder research. Front Cell Neurosci 10:215.

67. Shaltouki A, J Peng, Q Liu, MS Rao and X Zeng. (2013). Efficient generation of astrocytes from human pluripotent stem cells in defined conditions. Stem Cells 31:941-952.

68. Mormone E, S D'Sousa, V Alexeeva, MM Bederson and IM Germano. (2014). "Footprint-free", human induced pluripotent stem cell-derived astrocytes for in vivo cellbased therapy. Stem Cells Dev 23:2626-2636.

69. Emdad L, SL D'Souza, HP Kothari, Z a. Qadeer and IM Germano. (2012). Efficient differentiation of human embryonic and induced pluripotent stem cells into functional astrocytes. Stem Cells Dev 21:404-410.

70. Canfield SG, MJ Stebbins, BS Morales, SW Asai, GD Vatine, CN Svendsen, SP Palecek and EV Shusta. (2016). An isogenic blood-brain barrier model comprising brain endothelial cells, astrocytes and neurons derived from human induced pluripotent stem cells. J Neurochem 140: 874-888.

71. Yamamizu K, M Iwasaki, H Takakubo, T Sakamoto, T Ikuno, M Miyoshi, T Kondo, Y Nakao, M Nakagawa, H Inoue and JK Yamashita. (2017). In vitro modeling of blood-brain barrier with human iPSC-derived endothelial cells, pericytes, neurons, and astrocytes via notch signaling. Stem Cell Reports 8:1-14.

72. Yurugi-Kobayashi T, H Itoh, T Schroeder, A Nakano, G Narazaki, F Kita, K Yanagi, M Hiraoka-Kanie, E Inoue, et al. (2006). Adrenomedullin/cyclic AMP pathway induces notch activation and differentiation of arterial endothelial cells from vascular progenitors. Arterioscler Thromb Vasc Biol 26:1977-1984.

73. Yamamizu K, K Kawasaki, S Katayama, T Watabe and JK Yamashita. (2009). Enhancement of vascular progenitor potential by protein kinase A through dual induction of Flk-1 and Neuropilin-1. Blood 114:3707-3716.

74. Yamamizu K, T Matsunaga, S Katayama, H Kataoka, N Takayama, K Eto, S-I Nishikawa and JK Yamashita. (2012). PKA/CREB signaling triggers initiation of endothelial and hematopoietic cell differentiation via Etv2 induction. Stem Cells 30:687-696.

75. Yamamizu K, M Fujihara, M Tachibana, S Katayama, A Takahashi, E Hara, H Imai, Y Shinkai and JK Yamashita. (2012). Protein kinase A determines timing of early differentiation through epigenetic regulation with G9a. Cell Stem Cell 10:759-770.
76. Canfield SG, MJ Stebbins, HK Wilson, EV Shusta, SP Palecek and T Qian. (2016). Differentiation and characterization of human pluripotent stem cell-derived brain microvascular endothelial cells. Methods 101:93-102.

77. Page S, A Munsell and AJ Al-Ahmad. (2016). Cerebral hypoxia/ischemia selectively disrupts tight junctions complexes in stem cell-derived human brain microvascular endothelial cells. Fluids Barriers CNS 13:461.

78. Cecchelli R, C Coisne, S Aday, C Almeida, L Ferreira, M Culot, L Dehouck, M-P Dehouck, B Engelhardt and E Sevin. (2014). A stable and reproducible human bloodbrain barrier model derived from hematopoietic stem cells. PLoS One 9:e99733.

79. Audus KL and M Gumbleton. (2001). Progress and limitations in the use of in vitro cell cultures to serve as a permeability screen for the blood-brain barrier. J Pharm Sci 90:1681-1698.

80. Hall VJ. (2016). Modelling neurodegenerative diseases using human pluripotent stem cells. In: Pluripotent Stem Cells-From the Bench to the Clinic. Tomizawa M, ed. InTech, Rijeka, Croatia, pp 273-305.

81. Dimos JT, KT Rodolfa, KK Niakan, LM Weisenthal, H Mitsumoto, W Chung, GF Croft, G Saphier, R Leibel, et al. (2008). Induced pluripotent stem cells generated from patients with ALS can be differentiated into motor neurons. Science 321:1218-1221.

82. Kovacs GG. (2016). Molecular pathological classification of neurodegenerative diseases: turning towards precision medicine. Int J Mol Sci 17:189.

83. Park I-H, N Arora, H Huo, N Maherali, T Ahfeldt, A Shimamura, MW Lensch, C Cowan, K Hochedlinger and GQ Daley. (2008). Disease-specific induced pluripotent stem cells. Cell 134:877-886.

84. Tiscornia G, EL Vivas and JCI Belmonte. (2011). Diseases in a dish: modeling human genetic disorders using induced pluripotent cells. Nat Med 17:1570-1576.

85. Byers B, JP Cooke, A De, HN Nguyen, M Rollins, JC Lee, NF Huang, S Jamé, SS Gambhir, et al. (2011). Endothelial cells derived from human iPSCS increase capillary density and improve perfusion in a mouse model of peripheral arterial disease. Arterioscler Thromb Vasc Biol 31:e72-e79.

86. Nguyen HN, B Byers, B Cord, A Shcheglovitov, J Byrne, P Gujar, K Kee, B Schüle, RE Dolmetsch, et al. (2011). LRRK2 mutant iPSC-derived DA neurons demonstrate increased susceptibility to oxidative stress. Cell Stem Cell 8:267-280.

87. Reinhardt P, B Schmid, LF Burbulla, DC Schöndorf, L Wagner, M Glatza, S Höing, G Hargus, SA Heck, et al. (2013). Genetic correction of a LRRK2 mutation in human iPSCs links parkinsonian neurodegeneration to ERK-dependent changes in gene expression. Cell Stem Cell 12:354-367.

88. Chang K-H, G-J Lee-Chen, Y-R Wu, Y-J Chen, J-L Lin, M Li, I-C Chen, Y-S Lo, H-C Wu and C-M Chen. (2016). Impairment of proteasome and anti-oxidative pathways in the induced pluripotent stem cell model for sporadic Parkinson's disease. Parkinsonism Relat Disord 24:81-88.

89. Yagi T, D Ito, Y Okada, W Akamatsu, Y Nihei, T Yoshizaki, S Yamanaka, H Okano and N Suzuki. (2011). Modeling familial Alzheimer's disease with induced pluripotent stem cells. Hum Mol Genet 20:4530-4539.

90. Israel MA, SH Yuan, C Bardy, SM Reyna, Y Mu, C Herrera, MP Hefferan, S Van Gorp, KL Nazor, et al. (2012). Probing sporadic and familial Alzheimer's disease using induced pluripotent stem cells. Nature 482:216-220. 
91. Consortium THD iPSC. (2012). Induced pluripotent stem cells from patients with Huntington's disease show CAGrepeat-expansion-associated phenotypes. Cell Stem Cell 11:264-278.

92. Choi SH, YH Kim, M Hebisch, C Sliwinski, S Lee, C D’Avanzo, H Chen, B Hooli, C Asselin, et al. (2014). A three-dimensional human neural cell culture model of Alzheimer's disease. Nature 515:274-278.

93. Espuny-Camacho I, AM Arranz, M Fiers, A Snellinx, K Ando, S Munck, J Bonnefont, L Lambot, N Corthout, et al. (2017). Hallmarks of Alzheimer's disease in stemcell-derived human neurons transplanted into mouse brain. Neuron 93:1066-1081.e8.

94. Kortekaas R, KL Leenders, JCH Van Oostrom, W Vaalburg, J Bart, ATM Willemsen and NH Hendrikse. (2005). Blood-brain barrier dysfunction in Parkinsonian midbrain in vivo. Ann Neurol 57:176-179.

95. Bell RD, EA Winkler, I Singh, AP Sagare and R Deane. (2012). Apolipoprotein E controls cerebrovascular integrity via cyclophilin A. Nature 495:512-516.

96. Lacher SE, K Skagen, J Veit, R Dalton and EL Woodahl. (2015). P-glycoprotein transport of neurotoxic pesticides. J Pharmacol Exp Ther 355:99-107.

97. Desai BS, AJ Monahan, B Hendey and PM Carvey. (2007). Blood-brain barrier pathology in Alzheimer's and Parkinson's disease: implications for drug therapy. Cell Transplant 16:285-299.

98. Freude K, C Pires, P Hyttel and VJ Hall. (2014). Induced pluripotent stem cells derived from Alzheimer's disease patients: the promise, the hope and the path ahead. J Clin Med 3:1402-1436.

99. Winkler EA, Y Nishida, AP Sagare, SV Rege, RD Bell, D Perlmutter, JD Sengillo, S Hillman, P Kong, et al. (2015). GLUT1 reductions exacerbate Alzheimer's disease vasculoneuronal dysfunction and degeneration. Nat Neurosci 18: 521-530.

100. Broux B, A Prat and E Gowing. (2015). Glial regulation of the blood-brain barrier in health and disease. Semin Immunopathol 37:577-590.

101. Methia N, P André, A Hafezi-Moghadam, M Economopoulos, KL Thomas and DD Wagner. (2001). ApoE deficiency compromises the blood brain barrier especially after injury. Mol Med 7:810-815.

102. Hafezi-Moghadam A, KL Thomas and DD Wagner. (2007). ApoE deficiency leads to a progressive agedependent blood-brain barrier leakage. Am J Physiol Cell Physiol 292:C1256-C1262.

103. Alata W, Y Ye, I St-Amour, M Vandal and F Calon. (2015). Human apolipoprotein E $\varepsilon 4$ expression impairs cerebral vascularization and blood-brain barrier function in mice. J Cereb Blood Flow Metab 35:86-94.

104. Schofield R. (1978). The relationship between the spleen colony-forming cell and the haemopoietic stem cell. Blood Cells 4:7-25.

105. Scadden DT. (2006). The stem-cell niche as an entity of action. Nature 441:1075-1079.

106. Cosson S, EA Otte, H Hezaveh and JJ Cooper-White. (2015). Concise review: tailoring bioengineered scaffolds for stem cell applications in tissue engineering and regenerative medicine. Stem Cells Transl Med 4:156-164.

107. Yamada KM and E Cukierman. (2007). Modeling tissue morphogenesis and cancer in 3D. Cell 130:601-610.

108. Traub O and Berk BC. (1998). Laminar shear stress: mechanisms by which endothelial cells transduce an atheroprotective force. Arterioscler Thromb Vasc Biol Open Table Contents 18:677-685.

109. Ballermann BJ and MJ Ott. (1995). Adhesion and differentiation of endothelial cells by exposure to chronic shear stress: a vascular graft model. Blood Purif 13:125-134.

110. Naik P and L Cucullo. (2012). In vitro blood-brain barrier models: current and perspective technologies. J Pharm Sci 101:1337-1354.

111. Walsh TG, RP Murphy, P Fitzpatrick, KD Rochfort, AF Guinan, A Murphy and PM Cummins. (2011). Stabilization of brain microvascular endothelial barrier function by shear stress involves VE-cadherin signaling leading to modulation of pTyr-occludin levels. J Cell Physiol 226:3053-3063.

112. Booth R, S Noh and H Kim. (2014). A multiple-channel, multiple-assay platform for characterization of full-range shear stress effects on vascular endothelial cells. Lab Chip 14:1880-1890.

113. Cucullo L, M Hossain, V Puvenna, N Marchi and D Janigro. (2011). The role of shear stress in blood-brain barrier endothelial physiology. BMC Neurosci 12:40.

114. Takeshita Y, B Obermeier, A Cotleur, Y Sano, T Kanda and RM Ransohoff. (2014). An in vitro blood-brain barrier model combining shear stress and endothelial cell/astrocyte co-culture. J Neurosci Methods 232:165172 .

115. Huang H, Y Nakayama, K Qin, K Yamamoto, J Ando, J Yamashita, H Itoh, K Kanda, H Yaku, Y Okamoto and Y Nemoto. (2005). Differentiation from embryonic stem cells to vascular wall cells under in vitro pulsatile flow loading. J Artif Organs 8:110-118.

116. Santaguida S, D Janigro, M Hossain, E Oby, E Rapp and L Cucullo. (2006). Side by side comparison between dynamic versus static models of blood-brain barrier in vitro: a permeability study. Brain Res 1109:1-13.

117. Xu H, Z Zhuang, J Qin, G Zhu, Y Yu, M Zhang, WS Ho, F Yin, L Jiang, et al. (2016). A dynamic in vivo-like organotypic blood-brain barrier model to probe metastatic brain tumors. Sci Rep 6:36670.

118. Partyka PP, GA Godsey, JR Galie, MC Kosciuk, NK Acharya, RG Nagele and PA Galie. (2017). Mechanical stress regulates transport in a compliant $3 \mathrm{D}$ model of the blood-brain barrier. Biomaterials 115:30-39.

119. Booth R and H Kim. (2014). Permeability analysis of neuroactive drugs through a dynamic microfluidic in vitro blood-brain barrier model. Ann Biomed Eng 42:2379-2391.

120. Benton G, HK Kleinman, IP Arnaoutova and J George. (2009). Advancing science and technology via 3D culture on basement membrane matrix. J Cell Physiol 221:18-25.

121. Lancaster MA, ME Hurles, C-A Martin, AP Jackson, M Renner, D Wenzel, T Homfray, LS Bicknell, JM Penninger and JA Knoblich. (2013). Cerebral organoids model human brain development and microcephaly. Nature 501:373379.

122. Urich E, C Patsch, S Aigner, M Graf and R Iacone. (2013). Multicellular self-assembled spheroidal model of the blood brain barrier. Sci Rep 3:1500.

123. Gromnicova R, HA Davies, P Sreekanthreddy, IA Romero, T Lund, IM Roitt, JB Phillips, DK Male, H Wolburg, et al. (2013). Glucose-coated gold nanoparticles transfer across human brain endothelium and enter astrocytes in vitro. PLoS One 8:e81043.

124. Tourovskaia A, M Fauver and G Kramer. (2014). Tissueengineered microenvironment systems for modeling human vasculature. Exp Biol Med 239:264-1271. 
125. Neuhaus W, R Lauer, S Oelzant and UP Fringeli. (2006). A novel flow based hollow-fiber blood-brain barrier in vitro model with immortalised cell line PBMEC/C1-C2. J Biotechnol 125:127-141.

126. Cecchelli R, V Berezowski and S Lundquist. (2007). Modelling of the blood-brain barrier in drug discovery and development. Nat Rev Drug Discov 6:650-661.

127. Hossain M, W Tierney, L Cucullo and D Janigro. (2013). A new dynamic in vitro modular capillaries-venules modular system: cerebrovascular physiology in a box. BMC Neurosci 14:18.

128. van der Helm MW, AD van der Meer, JCT Eijkel, A van den Berg and LI Segerink. (2016). Microfluidic organ-onchip technology for blood-brain barrier research. Tissue Barriers 4:e1142493.

129. Hsieh F-Y, H-H Lin and S Hsu. (2015). 3D bioprinting of neural stem cell-laden thermoresponsive biodegradable polyurethane hydrogel and potential in central nervous system repair. Biomaterials 71:48-57.

130. Parekkadan B, Y Berdichevsky, D Irimia, A Leeder, G Yarmush, M Toner, JB Levine and ML Yarmush. (2008). Cell-cell interaction modulates neuroectodermal specification of embryonic stem cells. Neurosci Lett 438:190 195.

131. Lawrence BJ and SV Madihally. (2008). Cell colonization in degradable 3D porous matrices. Cell Adh Migr 2:9-16.

132. Chen G, Y Lv, P Guo, C Lin, X Zhang, L Yang and Z Xu. (2013). Matrix mechanics and fluid shear stress control stem cells fate in three dimensional microenvironment. Curr Stem Cell Res Ther 8:313-323.

133. Patel R and AJ Alahmad. (2016). Growth-factor reduced Matrigel source influences stem cell derived brain microvascular endothelial cell barrier properties. Fluids Barriers CNS 13:6.

134. Hopkins AM, E DeSimone, K Chwalek and DL Kaplan. (2015). 3D in vitro modeling of the central nervous system. Prog Neurobiol 125:1-25.

135. Al Ahmad A, CB Taboada, M Gassmann and OO Ogunshola. (2011). Astrocytes and pericytes differentially modulate blood-brain barrier characteristics during development and hypoxic insult. J Cereb Blood Flow Metab 31:693-705.

136. Hsieh F-Y and S Hsu. (2015). 3D bioprinting: a new insight into the therapeutic strategy of neural tissue regeneration. Organogenesis 11:153-158.

137. Mohanty S, J Trifol, M Alm, M Dufva, P Thomsen, M Hemmingsen, A Wolff, J Emnéus and A DolatshahiPirouz. (2016). 3D printed silicone-hydrogel scaffold with enhanced physicochemical properties. Biomacromolecules 17:1321-1329.

138. Ramirez F and DB Rifkin. (2003). Cell signaling events: a view from the matrix. Matrix Biol 22:101-107.

139. Zobel K, U Hansen and H-J Galla. (2016). Blood-brain barrier properties in vitro depend on composition and assembly of endogenous extracellular matrices. Cell Tissue Res 365:233-245.

140. Baeten KM and K Akassoglou. (2011). Extracellular matrix and matrix receptors in blood-brain barrier formation and stroke. Dev Neurobiol 71:1018-1039.

141. Wilson HK, SG Canfield, MK Hjortness, SP Palecek and EV Shusta. (2015). Exploring the effects of cell seeding density on the differentiation of human pluripotent stem cells to brain microvascular endothelial cells. Fluids Barriers CNS 12:13.

142. Blocki A, Y Wang, M Koch and P Peh. (2013). Not all MSCs can act as pericytes: functional in vitro assays to distinguish pericytes from other mesenchymal stem cells in angiogenesis. Stem Cells Dev 22:2347-2355.

143. Marion RM, K Strati, H Li, A Tejera, S Schoeftner, S Ortega, M Serrano and MA Blasco. (2009). Telomeres acquire embryonic stem cell characteristics in induced pluripotent stem cells. Cell Stem Cell 4:141-154.

144. Agarwal S, Y-H Loh, EM McLoughlin, J Huang, I-H Park, JD Miller, H Huo, M Okuka, RM dos Reis, et al. (2010). Telomere elongation in induced pluripotent stem cells from dyskeratosis congenita patients. Nature 464:292-296.

145. Prigione A, R Lurz, H Lehrach, B Fauler and J Adjaye. (2010). The senescence-related mitochondrial/oxidative stress pathway is repressed in human induced pluripotent stem cells. Stem Cells 28:721-733.

146. Studer L, E Vera and D Cornacchia. (2015). Programming and reprogramming cellular age in the era of induced pluripotency. Cell Stem Cell 16:591-600.

147. Miller JD, YM Ganat, S Kishinevsky, RL Bowman, B Liu, EY Tu, PK Mandal, E Vera, J Shim, et al. (2013). Human iPSC-based modeling of late-onset disease via progerininduced aging. Cell Stem Cell 13:691-705.

148. Poller B, H Gutmann, S Krähenbühl, B Weksler, I Romero, P-O Couraud, G Tuffin, J Drewe and J Huwyler. (2008). The human brain endothelial cell line hCMEC/D3 as a human blood-brain barrier model for drug transport studies. J Neurochem 107:1358-1368.

Address correspondence to: Karin Lauschke National Food Institute Technical University of Denmark Kemitorvet 202 Kongens Lyngby 2800 Denmark

E-mail: karla@food.dtu.dk

Received for publication January 5, 2017

Accepted after revision April 7, 2017 Prepublished on Liebert Instant Online April 11, 2017 